\title{
Random Phase Approximation Applied to Many-Body Noncovalent Systems
}

\author{
Marcin Modrzejewski, ${ }^{*}, \dagger$ Sirous Yourdkhani, ${ }^{\dagger}$ and Jiří Klimeš ${ }^{\dagger}$ \\ $\dagger$ Department of Chemical Physics and Optics, Faculty of Mathematics and Physics, Charles \\ University, Ke Karlovu 3, CZ-12116 Prague 2, Czech Republic \\ $\ddagger$ Faculty of Chemistry, University of Warsaw, Pasteura 1, 02-093 Warsaw, Poland
}

\section{Abstract}

The random phase approximation (RPA) has received a considerable interest in the field of modeling systems where noncovalent interactions are important. Its advantages over widely used density functional theory (DFT) approximations are the exact treatment of exchange and the description of long-range correlation. In this work we address two open questions related to RPA. First, how accurately RPA describes nonadditive interactions encountered in many-body expansion of a binding energy. We consider three-body nonadditive energies in molecular and atomic clusters. Second, how does the accuracy of RPA depend on input provided by different DFT models, without resorting to selfconsistent RPA procedure which is currently impractical for calculations employing periodic boundary conditions. We find that RPA based on the SCAN0 and PBE0 models, i.e., hybrid DFT, achieves an overall accuracy between CCSD and MP3 on a dataset of molecular trimers of Řezáč et al. (J. Chem. Theory. Comput. 2015, 11, 3065) Finally, many-body expansion for molecular clusters and solids often leads to a large number of small contributions that need to be calculated with a high precision. We therefore present a cubic-scaling (or SCF-like) implementation of RPA in atomic basis set, which is designed for calculations with a high numerical precision.

\section{Introduction}

Molecular solids are materials which are held by noncovalent interactions. Such molecular solids have often different phases or polymorphs that differ very little in energy. ${ }^{1,2}$ For example, in more than one half of the polymorph pairs studied by Nyman and Day a lattice energy difference was found to be less than $0.5 \mathrm{kcal} / \mathrm{mol}^{2}{ }^{2}$ There is an ongoing effort to develop theoretical methods that would reliably describe such minute differences. ${ }^{3-6}$ When the binding energy of a solid or a molecular cluster is decomposed using many-body expansion, the twobody contribution, corresponding to the binding of dimers, is the largest and as such it receives most of the attention in method development. However, nonadditive higher-order contributions, such as three-body and four-body terms can be important as well. For example, 3-body correlation effects represent some 5 to 7 $\%$ of the correlation contribution to the lattice energy in benzene. ${ }^{7,8}$ Moreover, they can affect the relative stability of different molecular clusters and solid phases. ${ }^{9-12}$

Kohn-Sham density functional theory (DFT) has been widely used to understand the binding of molecular solids or clusters. The missing description of long-range correlation (dispersion) has been viewed as the largest deficiency of approximate DFT for such systems. Improving the description of dispersion has therefore been one of most active fields of DFT development in the past decade. ${ }^{13-18}$ However, er- 
rors originating from the exchange functional are on the same order as the errors originating from the missing dispersion energy. ${ }^{19-21} \mathrm{Ap}$ proximate exchange functionals alone can lead to both strongly overestimated and underestimated noncovalent interactions. ${ }^{22}$ For two body systems such issues tend to be masked by adjusting the equilibrium- and short-distance behavior of the dispersion correction. ${ }^{19}$ However, the three-body exchange errors cannot be compensated in a similar way by adjusting the pairwise additive dispersion corrections. ${ }^{19}$ Overall, the conclusion originating from the existing literature is that no existing semilocal functional can reliably account for many body effects. ${ }^{21,23}$

Affordable schemes based on perturbation theory could offer higher and systematically improvable accuracy for calculations of condensed systems compared to standard DFT functionals. ${ }^{21,24-27}$ Of such schemes, the random phase approximation to the correlation energy (RPA) is promising as it is both compatible with the Hartree-Fock (HF) exchange and it contains terms describing higher-order (nonadditive) correlation effects. ${ }^{28,29}$ RPA has been tested for interaction energies of dimers, ${ }^{30-32}$ for adsorption, ${ }^{33-35}$ or for molecular ${ }^{36-39}$ and atomic solids ${ }^{40}$ and interfaces. ${ }^{41,42}$ For the cases involving noncovalent interactions, high accuracy has been achieved with addition of the singles corrections. ${ }^{43,44}$ However, its accuracy for predicting nonadditive energies is unknown and this is one of our interests in this work. Moreover, most of the RPA calculations nowadays are performed non-self-consistently, using DFT orbitals and energies in the RPA energy expression. Here we obtain RPA results using different DFT input orbitals. This allows us to understand if and how the errors in the underlying Kohn-Sham DFT potential transfer to the RPA results.

The three- and higher-body energies per single trimer are often small, on the order of microhartree or cal/mol or even less for larger distances. However, in molecular solids such contributions might not be negligible as there can be a large number of them. Therefore, they need to be evaluated with a high precision so that the resulting value is meaningful. To this end, we introduce an algorithm for calculating RPA correlation energies which uses Cholesky decomposition of the Coulomb operator matrix in atomic basis set together with eigendecomposition of the dielectric matrix. These steps lead to cubic scaling with the system size and to high and controllable precision. The details of the algorithm are presented in Section 2 followed by results on dimers and trimers of noble gases and results of the 3B-69 test set of Řezáč and coworkers. ${ }^{45}$

\section{Theory}

\section{$2.1 \quad$ Direct RPA}

The adiabatic-connection fluctuation-dissipation formula ${ }^{28,29}$ expresses the exact DFT correlation energy in terms of the density response function $\chi$ integrated over imaginary frequencies and the adiabatic connection coupling constant $\alpha$ :

$$
\begin{array}{r}
E_{\mathrm{c}}=-\frac{1}{2 \pi} \int_{0}^{1} \mathrm{~d} \alpha \int_{0}^{\infty} \mathrm{d} u \int \mathrm{d}^{3} \mathbf{r} \mathrm{d}^{3} \mathbf{r}^{\prime}\left(\chi^{\alpha}\left(\mathbf{r}, \mathbf{r}^{\prime} ; \mathrm{i} u\right)\right. \\
\left.-\chi^{0}\left(\mathbf{r}, \mathbf{r}^{\prime} ; \mathrm{i} u\right)\right) v_{\text {ee }}\left(\left|\mathbf{r}^{\prime}-\mathbf{r}\right|\right)
\end{array}
$$

Here, $v_{\mathrm{ee}}$ is the electron-electron interaction operator. The density response function produces a change in the electron density corresponding to a perturbation of the system's hamiltonian. For the noninteracting system at $\alpha=0$

$$
\delta \rho=\chi^{0} \delta v_{s}
$$

where $\delta v_{s}$ is the change in the Kohn-Sham effective potential. ${ }^{46}$ In this work, the density response function of the Kohn-Sham system is built using orbitals and orbital energies computed with an approximate exchangecorrelation functional, e.g., the Perdew, Burke, and Ernzerhof (PBE) functional ${ }^{47}$ and SCAN. ${ }^{48}$ An alternative, not explored here, would be to compute self-consistent orbitals. ${ }^{31,46,49,50}$ In either case, the formula for 
the density response reads

$$
\chi^{0}\left(\mathbf{r}, \mathbf{r}^{\prime} ; \mathrm{i} u\right)=2 \sum_{i a} \frac{\phi_{i}(\mathbf{r}) \phi_{a}(\mathbf{r}) \phi_{i}\left(\mathbf{r}^{\prime}\right) \phi_{a}\left(\mathbf{r}^{\prime}\right)}{\epsilon_{i}-\epsilon_{a}+\mathrm{i} u}+\text { c.c. }
$$

The factor of 2 originates from the spin summation over doubly occupied orbitals. Spin indices should be added for an open shell system. Throughout this work, we assume real orbitals expanded in an atomic-orbital basis set. Occupied and virtual indices are $i$ and $a$, respectively. The atomic orbital labels are $p, q, r$, and $s$.

The interacting density response at $\alpha>0$ is related to the Kohn-Sham response by the Dyson-type screening equation ${ }^{51}$

$$
\chi^{\alpha}=\chi^{0}+\chi^{0}\left(\alpha v_{\mathrm{ee}}+f_{\mathrm{xc}}^{\alpha}\right) \chi^{\alpha},
$$

where $f_{\mathrm{xc}}^{\alpha}$ is the frequency-dependent exchangecorrelation kernel. The direct random-phase approximation amounts to setting $f_{\mathrm{xc}}^{\alpha}=0$, which leads to

$$
\chi^{\alpha}=\left(1-\alpha \chi^{0} v_{\mathrm{ee}}\right)^{-1} \chi^{0} .
$$

We refer to direct RPA as "RPA" for short. As a consequence of the simple dependence on $\alpha$, the integration over the coupling constant in the ACFD formula is done analytically, which yields the final form of the RPA correlation energy

$$
E_{\mathrm{c}}=\frac{1}{2 \pi} \int_{0}^{\infty} \operatorname{Tr}\left(\ln \left(1-\chi^{0} v_{\mathrm{ee}}\right)+\chi^{0} v_{\mathrm{ee}}\right) \mathrm{d} u .
$$

To obtain a workable expression for the correlation energy, we define an auxiliary matrix $\boldsymbol{\Pi}(u)$, which is a symmetrized matrix representation of the product $-\chi^{0} v_{\text {ee }}$. With that definition, the energy expression reads

$$
E_{\mathrm{c}}=\frac{1}{2 \pi} \int_{0}^{\infty} \operatorname{Tr}(\ln (1+\Pi(u))-\Pi(u)) \mathrm{d} u .
$$

and is in practice evaluated with a numerical quadrature on a frequency grid.

\subsection{Effective basis for the RPA energy}

We now discuss the technical aspects of our implementation of the ACFD energy formula. The expression for $\chi^{0}$, Eq. 3, suggests that it can be represented using a basis of occupiedvirtual orbital pairs $\phi_{i}(\mathbf{r}) \phi_{a}(\mathbf{r})$. In canonicalbasis implementations ${ }^{52}$ the resulting matrix $\chi_{a i, b j}^{0}$ has a dimension of $N_{\text {occ }} N_{\text {virt }}$, implying a significant computational and storage cost beyond traditional DFT. To overcome that issue we use an alternative strategy which combines several algorithms to reduce the computational cost while not significantly reducing the precision of the result. First, we represent $\chi^{0}$ in the basis of atomic orbitals. This allows us to use the Laplace transform to calculate $\chi^{0}$ using Green's functions in imaginary time. Second, we use Cholesky decomposition of the Coulomb matrix to avoid the use of four-index Coulomb integrals. Finally, we approximate the auxiliary matrix $\boldsymbol{\Pi}$ by using only its dominant eigenvectors. These steps allow for a cubic scaling implementation and a well-defined control of precision.

Let us first write down the Kohn-Sham density response function as

$$
\begin{aligned}
& \chi^{0}\left(\mathbf{r}, \mathbf{r}^{\prime} ; i u\right)= \\
& \sum_{i a} \phi_{i}(\mathbf{r}) \phi_{a}(\mathbf{r}) \frac{4\left(\epsilon_{i}-\epsilon_{a}\right)}{\left(\epsilon_{i}-\epsilon_{a}\right)^{2}+u^{2}} \phi_{i}\left(\mathbf{r}^{\prime}\right) \phi_{a}\left(\mathbf{r}^{\prime}\right) \\
& \quad=-4 \sum_{i a} \phi_{i}(\mathbf{r}) \phi_{a}(\mathbf{r}) \frac{d_{a i}}{d_{a i}^{2}+u^{2}} \phi_{i}\left(\mathbf{r}^{\prime}\right) \phi_{a}\left(\mathbf{r}^{\prime}\right)
\end{aligned}
$$

where

$$
d_{a i}=\epsilon_{a}^{\prime}-\epsilon_{i}^{\prime}
$$

and the orbital energies $\epsilon_{a}^{\prime}=\epsilon_{a}-\epsilon_{\mathrm{F}}$ and $\epsilon_{i}^{\prime}=$ $\epsilon_{i}-\epsilon_{\mathrm{F}}$ are shifted by the Fermi energy $\epsilon_{\mathrm{F}}=$ $\left(\epsilon_{\mathrm{HOMO}}+\epsilon_{\mathrm{LUMO}}\right) / 2$. Following Kaltak et al., ${ }^{53}$ we separate the occupied and virtual indices by applying the Laplace transform

$$
\begin{aligned}
\frac{d_{a i}}{d_{a i}^{2}+u^{2}} & =\int_{0}^{\infty} \cos (u t) \exp \left(-d_{a i} t\right) \mathrm{d} t \\
& =\int_{0}^{\infty} \cos (u t) \exp \left(-\epsilon_{a}^{\prime} t\right) \exp \left(\epsilon_{i}^{\prime} t\right) \mathrm{d} t
\end{aligned}
$$


Now, expanding the molecular orbitals in terms of atomic functions

$$
\begin{aligned}
& \phi_{i}(\mathbf{r})=\sum_{p} C_{p i} \phi_{p}(\mathbf{r}) \\
& \phi_{a}(\mathbf{r})=\sum_{p} C_{p a} \phi_{p}(\mathbf{r})
\end{aligned}
$$

yields the density response function expressed completely in terms of $\mathrm{AO}$ indices

$$
\chi_{p q, r s}^{0}(\mathrm{i} u)=-4 \int_{0}^{\infty} \cos (u t) \rho_{p r}^{\mathrm{occ}}(t) \rho_{q s}^{\mathrm{virt}}(t) \mathrm{d} t
$$

The matrices $\rho^{\text {occ }}$ and $\rho^{\text {virt }}$ are hole and particle noninteracting Green's functions at imaginary time

$$
\begin{aligned}
\rho_{p q}^{\text {occ }}(t) & =\sum_{i} C_{p i} \exp \left(\epsilon_{i}^{\prime} t\right) C_{q i} \\
\rho_{p q}^{\text {virt }}(t) & =\sum_{a} C_{p a} \exp \left(-\epsilon_{a}^{\prime} t\right) C_{q a} .
\end{aligned}
$$

In principle, the summations over $\mathrm{AO}$ pairs $p q$ run over a range much larger than the original set of occupied-virtual pairs $i a$. However, in a Gaussian orbital basis set, the Coulomb matrix elements $V_{p q, r s}=\left(p q\left|v_{\text {ee }}\right| r s\right)$ acquire Gaussian damping factors which decay quickly with the distance between the centers of $p q$ and $r s$. To take advantage of that, we constrain the computations and storage to the set of significant orbital shell pairs $\mathcal{S}$. Instead of full summations over $p q$, all sums traverse $\mathcal{O}(N)$ elements of $\mathcal{S}$. The set $\mathcal{S}$ is constructed by discarding small diagonal Coulomb integrals as long as the trace error of the Coulomb matrix lies below a predefined error bound

$$
\sum_{p q} V_{p q, p q}-\sum_{p q \in \mathcal{S}} V_{p q, p q}<\tau_{\text {screen }} .
$$

(Note that the bookkeeping is done for whole shell pairs and not for the individual angular functions.) The prescreening is mostly effective for medium and large systems, which includes noncovalent complexes of small molecules at separations beyond equilibrium. For example, in an RPA/aug-cc-pVQZ calculation for methane in a $\left(\mathrm{H}_{2} \mathrm{O}\right)_{20}$ cage, the prescreening subroutine discards $60 \%$ of the $6.9 \times 10^{6}$ orbital pairs at the default level of numerical precision.
We have found that the screening based on the $p q$ and $r s$ AO pairs is compatible with high precision targets for the total energy (i.e., with the target error on the order of $10^{-5} \mathrm{kcal} / \mathrm{mol}$ for the interaction energy).

Multiple techniques exploit the redundancy in the matrix $\boldsymbol{V}$ to avoid dealing with fourindex Coulomb integrals. Eshuis et al. ${ }^{54}$ and Ren et al. ${ }^{55}$ have applied density fitting with the Coulomb metric in their quartic-scaling RPA implementations. Wilhelm et al. ${ }^{56}$ and Schurkus and Ochsenfeld ${ }^{57}$ have achieved cubic and linear scaling, respectively, owing to the sparse matrices appearing in density fitting based on the overlap metric. While using the overlap metric introduces sparsity, it is orders of magnitude less precise than fitting with the Coulomb metric or Cholesky decomposition. The sensitivity of the RPA energy to different choices of the fitting metric is investigated in Refs. 58 and 55 .

We have found that for our applications, which require extremely precise interaction energies, the most dependable method of decomposing the Coulomb matrix is the pivoted Cholesky decomposition ${ }^{59,60}$ in which the Coulomb matrix is written as

$$
V_{p q, r s}=\sum_{k}^{N_{\text {Chol }}} R_{p q, k} R_{r s, k} \quad \text { for } p q, r s \in \mathcal{S} .
$$

The number of Cholesky vectors $N_{\text {Chol }}$ is increased until the Cholesky vectors matrix $\boldsymbol{R}$ satisfies the Coulomb matrix trace condition ${ }^{60}$

$$
\operatorname{Tr}(\boldsymbol{V})-\operatorname{Tr}\left(\boldsymbol{R} \boldsymbol{R}^{T}\right)<\tau_{\text {Chol }}
$$

Unlike density fitting, the Cholesky decomposition of $\boldsymbol{V}$ automatically achieves an arbitrary precision level within the machine limits, without the need for supplying a predefined auxiliary basis set. That is in line with the requirement for extra numerical precision for $n$-body noncovalent energies. ${ }^{61}$ In practice, we apply Eq. 18 with a wide margin of safety to ensure that the Coulomb matrix decomposition does not contribute to the overall numerical error. (See Table 1 for the threshold values.) As a consequence our computations involve the number 
of Cholesky vectors which is significantly larger than in typical calculations involving the decomposition of the Coulomb integrals. For example, for water dimer in equilibrium geometry, the number of Cholesky vectors is six times the number of atomic orbitals. However, this does not affect significantly the cost of the RPA program as the Cholesky vectors are further contracted into a much more compact basis spanning the dominant eigenspace of $\boldsymbol{\Pi}$, as discussed later in the text.

We employ the Cholesky algorithm described in Ref. 59 with the following modifications: (i) all AO pair indices belong to the set $\mathcal{S}$ defined in Eq. 16, (ii) the convergence condition involves the trace error of the Coulomb matrix. In contrast to the usual condition of the minimum decomposed diagonal element, the condition given in Eq. 18 leads to extra numerical precision in absolute energies, and thus less reliance on error cancellation for energy differences.

Having the Cholesky-decomposed Coulomb matrix, we now use it to rewrite the expression for the correlation energy. To this end we consider the lowest order term in Eq. 6. In matrix form one can write

$$
\begin{aligned}
& \operatorname{Tr}\left(\chi^{0}(u) v_{\mathrm{ee}}\right)=\sum_{p q, r s \in \mathcal{S}} \chi_{p q, r s}^{0}(u) V_{r s, p q} \\
& =\operatorname{Tr}\left(\chi^{0}(u) \boldsymbol{R} \boldsymbol{R}^{T}\right)=\operatorname{Tr}\left(\boldsymbol{R}^{T} \chi^{0}(u) \boldsymbol{R}\right) .
\end{aligned}
$$

The formula is analogous to the one derived by Ren et al. ${ }^{55}$, except for the use of the Cholesky vectors instead of a one-center auxiliary basis set. We use the last expression of Eq. 19 to define the auxiliary matrix $\boldsymbol{\Pi}$ in the Cholesky basis

$$
\Pi(u)=-\boldsymbol{R}^{T} \chi^{0}(u) \boldsymbol{R} .
$$

One can see that the dimension of $\boldsymbol{\Pi}(u)$ is given by the number of Cholesky vectors $N_{\text {Chol }} \sim$ $\mathcal{O}(N)$ instead of the usual $N_{\text {occ }} N_{\text {virt }}$. Our RPA program stores and computes only the matrix elements corresponding to $p \geq q$ and $p q \in \mathcal{S}$. We also halve the cost of the dominant step, i.e., the matrix multiplication $\chi^{0}(u) \boldsymbol{R}$, by utilizing the permutational symmetry $\chi_{p q, r s}^{0}=\chi_{r s, p q}^{0}$.

At this point one could diagonalize or LU- decompose $\boldsymbol{\Pi}(u)$ to obtain its eigenvalues and hence the correlation energy. Remarkably, a further reduction of the computational cost is possible at a given precision level without additional assumptions on the sparsity of $\rho^{\text {occ }}$ and $\rho^{\text {virt }}$. As shown by Galli et al., ${ }^{62-64}$ the $\mathrm{RPA}$ dielectric matrix ${ }^{46} \epsilon_{\mathrm{RPA}}=1-v_{\mathrm{ee}} \chi^{0}$, which is closely related to $\boldsymbol{\Pi}$ defined in this work, can be accurately reconstructed from a small subset of its most heavily screened eigenpotentials. That idea has been employed by Nguyen and de Gironcoli ${ }^{65}$ to compute the RPA correlation energy using the eigenpotentials of $\chi^{0}$ obtained with first-order density-functional perturbation theory (DFPT). ${ }^{66}$ In their planewave/pseudopotential code, ${ }^{67}$ which scales as $\mathcal{O}\left(N^{4}\right)$, the authors of Ref. 65 solve the perturbed Kohn-Sham equations for the density perturbation $\delta \rho$, which is the result of $\chi^{0}$ acting on a trial eigenpotential. A repeated insertion of trial potentials into the first-order DFPT equations refines the guess eigenvectors of $\chi^{0}$ for each frequency of the ACFD integral, without a summation over virtual states. In a recent work, Hellgren et al. ${ }^{68}$ have demonstrated the viability of that approach for RPAX calculations for noncovalent dimers. (See also Ref. 69 for details of the projective eigendecomposition of the dielectric screening method in the GW calculations.)

Here, we introduce an AO basis method of computing $E_{\mathrm{c}}$ by projecting $\boldsymbol{\Pi}$ onto its dominant eigenvectors. The novelty of our approach lies in combining the eigendecomposition of $\boldsymbol{\Pi}$ with a cubic scaling AO method based on the Cholesky decomposition.

Let the eigenvalues of the positive-definite matrix $\Pi(u)$ be

$$
\lambda_{1} \geq \lambda_{2} \geq \ldots \geq \lambda_{N_{\text {Chol }}} \geq 0 .
$$

We take a subset of $N_{\text {eig }}$, with $N_{\text {eig }} \leq N_{\text {Chol }}$, largest eigenvalues $\lambda_{1}, \lambda_{2}, \ldots, \lambda_{N_{\text {eig }}}$ and store them in a matrix $\boldsymbol{G}$. The matrix $\boldsymbol{G}$ then transforms $\boldsymbol{\Pi}$ to its effective reduced-dimension form defined as

$$
\Pi^{\prime}=\boldsymbol{G}^{T} \Pi \boldsymbol{G}
$$

Given a user-defined threshold $\tau_{\text {trace }}$, we adjust $N_{\text {eig }}$ by appending $\boldsymbol{G}$ with new columns 
until the difference between the exact and the reduced-dimension traces satisfies

$$
0 \leq \operatorname{Tr}(\boldsymbol{\Pi})-\operatorname{Tr}\left(\boldsymbol{\Pi}^{\prime}\right)=\sum_{k=N_{\text {eig }}+1}^{N_{\text {Chol }}} \lambda_{k}<\tau_{\text {trace }}
$$

Importantly, the trace error in $\boldsymbol{\Pi}^{\prime}$ introduces a quadratic error per single frequency in the ACFD integral

$$
\begin{gathered}
\delta=\operatorname{Tr}(\ln (\mathbf{1}+\boldsymbol{\Pi}))-\operatorname{Tr}(\boldsymbol{\Pi}) \\
-\left(\operatorname{Tr}\left(\ln \left(\mathbf{1}+\boldsymbol{\Pi}^{\prime}\right)-\operatorname{Tr}\left(\boldsymbol{\Pi}^{\prime}\right)\right)\right. \\
=\left|\sum_{k=N_{\text {eig }}+1}^{N_{\text {Chol }}} \ln \left(1+\lambda_{k}\right)-\lambda_{k}\right| \leq\left|\sum_{k=N_{\text {eig }}+1}^{N_{\text {Chol }}} \frac{1}{2} \lambda_{k}^{2}\right| \\
\leq \frac{1}{2}\left|\sum_{k=N_{\text {eig }}+1}^{N_{\text {Chol }}} \lambda_{k}\right|^{2}<\frac{1}{2} \tau_{\text {trace }}^{2} .
\end{gathered}
$$

The RPA correlation energy expressed with the effective matrix $\boldsymbol{\Pi}^{\prime}$ is

$$
E_{\mathrm{c}}=\frac{1}{2 \pi} \int_{0}^{\infty} \operatorname{Tr}\left(\ln \left(1+\boldsymbol{\Pi}^{\prime}(u)\right)-\boldsymbol{\Pi}^{\prime}(u)\right) \mathrm{d} u .
$$

The trace error $\delta$ is summed up over about 10 20 points on the frequency grid. Nonetheless, as the energy contributions fall off steeply with increasing frequency, the trace error at a few lowest frequencies dominates the integrated error.

Of course there would be no computational gain if the vectors of $\boldsymbol{G}$ were calculated by exact diagonalization for $\Pi(u)$ at every frequency point. To solve the above issue, we compute $\boldsymbol{G}$ only once, for the lowest frequency of the numerical grid and use it for all other frequencies. To avoid the diagonalization of the fulldimension matrix $\boldsymbol{\Pi}$, we build the matrix $\boldsymbol{G}$ by applying a variant of the subspace iteration method starting from random guess vectors, as presented by Saibaba et al. ${ }^{70}$ Given the matrix $\boldsymbol{\Pi}$ for the lowest frequency, we carry out the iterations

$$
\begin{aligned}
\boldsymbol{G}^{[0]} & \leftarrow \text { random numbers from } \mathcal{N}(0,1) \\
\boldsymbol{G}^{[m]} & \leftarrow \text { QR decomposition of } \boldsymbol{\Pi} \boldsymbol{G}^{[m-1]} .
\end{aligned}
$$

As shown in Ref. 70, the trace error resulting from the subspace iteration method approaches the sum of neglected small eigenvalues exponentially fast with the number of iterations, which makes valid our error bound of Eq. 24. In all RPA calculations we used $m=2$ iterations to obtain $\boldsymbol{G}$. We observed only an insignificant difference in the number of basis vectors as compared to exact diagonalization. For water dimer in equilibrium configuration, the exact diagonalization yields 598 effective basis vectors, whereas with the subspace iteration approach the number is 603 .

The complete computational scheme involves both dielectric eigenvectors, i.e., the eigenvectors of $\boldsymbol{\Pi}$, and the Cholesky decomposition of the Coulomb matrix. To reduce the number of floating-point operations and storage requirements, we obtain $\boldsymbol{G}$ for the full-dimension $\boldsymbol{\Pi}$ at the lowest frequency and use it to build a new matrix

$$
\boldsymbol{R}^{\prime}=\boldsymbol{R} \boldsymbol{G}
$$

At this point we deallocate $\boldsymbol{R}$ as it is no longer needed. We subsequently reuse $\boldsymbol{R}^{\prime}$ to obtain the auxiliary matrix

$$
\boldsymbol{\Pi}^{\prime}(u)=-\boldsymbol{R}^{\prime T} \chi^{\mathbf{0}}(u) \boldsymbol{R}^{\prime}
$$

at all frequencies. With the screening condition of Eq. 16, the dimension of $\boldsymbol{R}^{\prime}$ is $N_{\mathrm{AO}} \times N_{\text {eig }}$. The most compute-intensive steps are the formation of $\chi^{\mathbf{0}}(u) \boldsymbol{R}^{\prime}$ (linear speed-up when using the dominant eigenspace) and the matrix multiplication $\boldsymbol{R}^{\prime T}\left(\chi^{\mathbf{0}}(u) \boldsymbol{R}^{\prime}\right)$ (quadratic speed-up). In parallel computations, the blocks of $\boldsymbol{R}^{\prime}$ are distributed between concurrent processes and each process builds its own chunk of $\chi^{\mathbf{0}}(u) \boldsymbol{R}^{\prime}$.

To get properly size-extensive interaction energy, we use the same effective eigenspace for the bound complex and for all its subsystems. For example, when calculating nonadditive three-body noncovalent interaction energies, we obtain the matrix $\boldsymbol{R}^{\prime}$ for a trimer $A B C$ and reuse it for dimers $A B, B C, A C$, as well as for monomers $A, B$, and $C$.

\subsection{Numerical integration}

In our implementation there are two integrals which need to be calculated numerically, similar to the implementation of Kaltak et al. ${ }^{53,71}$ First, 
the correlation energy is obtained by integration over an imaginary frequency grid. Second, an imaginary time grid is needed to represent Green's functions from which the response function is calculated via the Laplace transform.

The frequency integral of Eq. 6 is approximated as

$E_{\mathrm{c}}=\frac{1}{2 \pi} \sum_{k=1}^{n} w_{k n} \operatorname{Tr}\left[\ln \left(\mathbf{1}+\boldsymbol{\Pi}^{\prime}\left(u_{k n}\right)\right)-\boldsymbol{\Pi}^{\prime}\left(u_{k n}\right)\right]$,

where the frequencies $u_{k n}$ and weights $w_{k n}$ are given by the points $x_{k n}^{\mathrm{GL}}$ and weights $w_{k n}^{\mathrm{GL}}$ of an $n$-point Gauss-Legendre quadrature mapped onto the half-infinite interval

$$
\begin{aligned}
& u_{k n}(\zeta)=\zeta \frac{1+x_{k n}^{\mathrm{GL}}}{1-x_{k n}^{\mathrm{GL}}} \\
& w_{k n}(\zeta)=\zeta \frac{2 w_{k n}^{\mathrm{GL}}}{\left(1-x_{k n}^{\mathrm{GL}}\right)^{2}} .
\end{aligned}
$$

Moreover, we have modified the mapping used by Ren et al. ${ }^{55}$ by making the parameter $\zeta$ a variable adjusted per system. To find the optimal $\zeta$ we consider the frequency-dependent part of Eq. 8 as a test function. The exact integral of the test function and its quadrature approximation for a number of nodes $n$ are given as

$$
\begin{gathered}
\mathcal{I}_{\text {exact }}\left(d_{a i}\right)=\int_{0}^{\infty} \frac{\mathrm{d} u}{d_{a i}^{2}+u^{2}}=\frac{\pi}{2 d_{a i}} \\
\mathcal{I}_{\text {quad }}\left(d_{a i} ; \zeta, n\right)=\sum_{k=1}^{n} \frac{w_{k n}(\zeta)}{d_{a i}^{2}+u_{k n}(\zeta)^{2}} .
\end{gathered}
$$

The optimal $\zeta$ then minimizes the squared error averaged over the distribution of orbital energy differences

$$
\begin{aligned}
\zeta(n)=\underset{\zeta^{\prime}}{\arg \min } \max _{\mu} & \sum_{\nu} h\left(d_{\nu \mu}\right)\left(\mathcal{I}_{\text {exact }}\left(d_{\nu \mu}\right)\right. \\
& \left.-\mathcal{I}_{\text {quad }}\left(d_{\nu \mu} ; \zeta^{\prime}, n\right)\right)^{2} .
\end{aligned}
$$

The weighting function $h\left(d_{\nu \mu}\right)$ counts how many orbital energy differences fall into a histogram bin $\nu$ for a system $\mu$. The set of all energy differences is divided into 100 bins for each system. In the case of a trimer, the maximum in Eq. 35 is taken over the set of the trimer and all of the dimer and monomer subsystems so that the interacting noncovalent complex and all its subsystems share the quadrature points and weights. Owing to the common grid, the interaction energy includes only the physical interactions and not the effects of changing the grid between subsystems. In particular, at large separations, the interaction energy properly goes to zero. To be able to define the common grids the SCF for all subsystems must precede the RPA program. The number of nodes $n$ is the smallest integer which satisfies the root-mean square error and the maximum relative error conditions

$$
\begin{gathered}
\max _{\mu}\left(\sum _ { \nu } h ( d _ { \nu \mu } ) \left(\mathcal{I}_{\text {exact }}\left(d_{\nu \mu}\right)\right.\right. \\
\left.\left.-\mathcal{I}_{\text {quad }}\left(d_{\nu \mu} ; \zeta(n), n\right)\right)^{2}\right)^{1 / 2}<\tau_{\text {freq,RMSD }} \\
\max _{\mu \nu}\left|\frac{\mathcal{I}_{\text {exact }}\left(d_{\nu \mu}\right)-\mathcal{I}_{\text {quad }}\left(d_{\nu \mu} ; \zeta(n), n\right)}{\mathcal{I}_{\text {exact }}\left(d_{\nu \mu}\right)}\right| \\
<\tau_{\text {freq, MaxRel }}
\end{gathered}
$$

The imaginary time integral includes an oscillatory integrand depending on the frequency of the density response function

$$
\begin{aligned}
& \chi_{p q, r s}^{0}\left(\mathrm{i} u_{k n}\right) \\
& \quad=-4 \int_{0}^{\infty} \cos \left(u_{k n} t\right) \rho_{p r}^{\mathrm{occ}}(t) \rho_{q s}^{\mathrm{virt}}(t) \mathrm{d} t \\
& =-4 \sum_{l=1}^{n^{\prime}} w_{l n^{\prime}}^{\prime} \rho_{p r}^{\mathrm{occ}}\left(t_{l n^{\prime}}\right) \rho_{q s}^{\mathrm{virt}}\left(t_{l n^{\prime}}\right) .
\end{aligned}
$$

The parameters of the quadrature, $t_{l n^{\prime}}, w_{l n^{\prime}}^{\prime}$, and $n^{\prime}$, are adjusted for each $u_{k n}$. For nearzero frequencies, we use the minimax quadrature of Takatsuka et al., ${ }^{72}$ which is designed for decomposing the zero-frequency denominators occurring in Laplace-transformed MP2. ${ }^{73,74}$ For higher frequencies, we use the robust double exponential quadrature of Ooura and Mori. ${ }^{75}$ (See Eq. 4.2 in Ref. 75.) Using the test function and 
its quadrature approximation

$$
\begin{aligned}
\mathcal{I}_{\text {exact }}^{\prime}\left(d_{a i}, u_{k n}\right) & =\frac{d_{a i}}{d_{a i}^{2}+u_{k n}^{2}} \\
\mathcal{I}^{\prime}{ }_{\text {quad }}\left(d_{a i}, u_{k n} ; n^{\prime}\right) & =\sum_{l=1}^{n^{\prime}} w_{l n^{\prime}}^{\prime}\left(u_{k n}\right) \exp \left(-d_{a i} t_{l n^{\prime}}\left(u_{k n}\right)\right)
\end{aligned}
$$

we fix the number of grid points $n^{\prime}$ as the smallest integer satisfying both

$$
\begin{aligned}
& \max _{\mu}\left(\sum _ { \nu } h ( d _ { \nu \mu } ) \left(\mathcal{I}_{\text {exact }}^{\prime}\left(d_{\nu \mu}\right)\right.\right. \\
& \left.\left.-\mathcal{I}_{\text {quad }}^{\prime}\left(d_{\nu \mu} ; u_{k n}, n^{\prime}\right)\right)^{2}\right)^{1 / 2}<\tau_{\text {imag,RMSD }}
\end{aligned}
$$

and

$$
\begin{aligned}
& \max _{\mu \nu}\left|\frac{\mathcal{I}_{\text {exact }}^{\prime}\left(d_{\nu \mu}\right)-\mathcal{I}_{\text {quad }}^{\prime}\left(d_{\nu \mu} ; u_{k n}, n^{\prime}\right)}{\mathcal{I}_{\text {exact }}^{\prime}\left(d_{\nu \mu}\right)}\right| \\
& <\tau_{\text {imag,MaxRel }} \text {. }
\end{aligned}
$$

The transition point between the minimax and double exponential quadratures depends on which approach is able to satisfy the above conditions with a smaller number of points. Similarly to the frequency quadrature, the complex and all its subsystems share the same imaginary time grid.

The thresholds controlling the grid accuracy as well as other thresholds used for the RPA correlation energy are summarized in Table 1. The specified values are hand-tuned against accurate RPA calculations on our calibration set of noncovalent dimers and trimers. The data in Table 2 demonstrate the influence of the numerical settings on 2-body and 3-body interaction energies.

\subsection{Singles correction}

From the point of view of ordinary RayleighSchrodinger perturbation theory, the KohnSham orbitals are noncanonical and the correlation energy includes nonzero terms related to single excitations from the Kohn-Sham determinant. The usefulness of a beyond-RPA approach including singles was first demonstrated by Ren et al., ${ }^{43}$ who, in their study of noncova- lent interaction energies, employed the HartreeFock (HF) orbitals to compute the mean-field part of the RPA total energy and the KohnSham orbitals to obtain the correlation part. The renormalized singles formula which sums up singles through infinite order was later derived in Ref. 32. Finally, Klimes et al. ${ }^{44}$ derived the renormalized singles term in a form that is applied in this work

$$
\begin{aligned}
E_{\mathrm{c}}^{\mathrm{RSE}}=2 \operatorname{Tr} & \left(\boldsymbol{\rho}^{\mathrm{HF}} \boldsymbol{F}^{\mathrm{HF}}\left[\boldsymbol{\rho}^{\mathrm{DFT}}\right]\right) \\
& -2 \operatorname{Tr}\left(\boldsymbol{\rho}^{\mathrm{DFT}} \boldsymbol{F}^{\mathrm{HF}}\left[\boldsymbol{\rho}^{\mathrm{DFT}}\right]\right) .
\end{aligned}
$$

The self-consistent Kohn-Sham orbitals are used to build the density matrix $\boldsymbol{\rho}^{\mathrm{DFT}}$ and the HF hamiltonian $\boldsymbol{F}^{\mathrm{HF}}\left[\boldsymbol{\rho}^{\mathrm{DFT}}\right]$. In contrast to the approach presented in Ref. 43, here the density matrix $\boldsymbol{\rho}^{\mathrm{HF}}$ is obtained in a single step diagonalization from the eigenvectors of $\boldsymbol{F}^{\mathrm{HF}}\left[\boldsymbol{\rho}^{\mathrm{DFT}}\right]$. The computational cost of RSE equals that of a single Fock matrix evaluation. A higheraccuracy variant of the singles correction includes the density from the $G W$ method instead of $\boldsymbol{\rho}^{\mathrm{HF}} .{ }^{44}$

Adding the singles corrections has been shown to improve the RPA binding energies for several systems. ${ }^{43,44}$ However, how they affect threebody energies is unknown and this is one of our interests in this work. Another point we try to understand is how do the singles depend on the input DFT orbitals.

\section{Numerical results}

\subsection{Technical details}

In this study we use RPA and wavefunction methods to obtain two-body interaction energies

$$
E_{\mathrm{int}}=E(A B)-E(A)-E(B)
$$

and nonadditive three-body interaction energy components

$$
\begin{gathered}
E_{\text {int }}[3,3]=E(A B C)-E(A B)-E(B C) \\
-E(A C)+E(A)+E(B)+E(C)
\end{gathered}
$$


Table 1: Thresholds, in atomic units, controlling the numerical precision of the RPA correlation energy.

\begin{tabular}{llll}
\hline & 1 & 2 & 3 \\
\hline$\tau_{\text {screen }}$ & $10^{-6}$ & $10^{-7}$ & $10^{-8}$ \\
$\tau_{\text {Chol }}$ & $10^{-2}$ & $10^{-3}$ & $10^{-4}$ \\
$\tau_{\text {freq,RMSD }}$ & $10^{-6}$ & $10^{-6}$ & $10^{-6}$ \\
$\tau_{\text {freq,MaxRel }}$ & $10^{-3}$ & $10^{-3}$ & $10^{-3}$ \\
$\tau_{\text {imag,RMSD }}$ & $10^{-6}$ & $10^{-6}$ & $10^{-6}$ \\
$\tau_{\text {imag,MaxRel }}$ & $10^{-3}$ & $10^{-3}$ & $10^{-3}$ \\
$\tau_{\text {trace }}$ & $\sqrt{10^{-5}}$ & $\sqrt{10^{-7}}$ & $10^{-5}$ \\
\hline
\end{tabular}

Table 2: Numerical precision of the RPA correlation energies. The uppermost row contains the reference correlation parts of two- and threebody energies. The rows corresponding to the parameter sets 1 ...3 (defined in Table 1) contain the numerical errors with respect to the reference. All energies are in kcal/mol. The aug-cc-pVQZ basis is used for all datapoints. The spacing between atoms in the linear configuration is $R=3 \AA$. The neon trimer at $26.6^{\circ}$ is an isosceles triangle with the base $R=2.8 \AA$.

\begin{tabular}{lrrrrr}
\hline Numerical precision & $\mathrm{Ne}_{2}$ & $\left(\mathrm{NH}_{3}\right)_{2}$ & linear $\mathrm{Ne}_{3}$ & $\left(\mathrm{H}_{2} \mathrm{O}\right)_{2}$ & $\mathrm{Ne}_{3}\left(26.6^{\circ}\right)$ \\
\hline Reference & -0.167650 & -1.783236 & -0.0003748 & -1.320970 & 0.322301 \\
1 & $-4 \times 10^{-5}$ & $-3 \times 10^{-5}$ & $-3 \times 10^{-7}$ & $-5 \times 10^{-4}$ & $-1 \times 10^{-4}$ \\
2 & $-2 \times 10^{-5}$ & $3 \times 10^{-5}$ & $1 \times 10^{-7}$ & $3 \times 10^{-5}$ & $3 \times 10^{-6}$ \\
3 & $-6 \times 10^{-6}$ & $-5 \times 10^{-6}$ & $4 \times 10^{-8}$ & $-1 \times 10^{-5}$ & $4 \times 10^{-6}$ \\
\hline
\end{tabular}

In all calculations, the monomer, dimer, and trimer energies are computed in the trimer basis set and at the same geometries as in the trimer.

The RPA calculations were carried out using in-house software implementing the algorithms presented in Sections 2.2 and 2.3. The code also calculates the exact-exchange (EXX) component of the energy (the Hartree-Fock-like part of the RPA energy evaluated with DFT orbitals) and the singles correction. The Molpro pack$\operatorname{age}^{76}$ was used to obtain the HF energy and correlation energy at the coupled cluster level and using different orders of Møller-Plesset perturbation theory. ${ }^{77,78}$ Moreover, we used Molpro $^{76}$ to obtain three-body dispersion energies. Finally, VASP was used to obtain a GWSE correction for neon trimer. ${ }^{44,53,71,79}$

The correlation energies, including RPA, typically converge slowly with the basis set size. We therefore extrapolated all wavefunction and RPA correlation energies to complete basis-set
(CBS) limit $E_{\mathrm{CBS}}$ using the two-point scheme of Halkier et al. ${ }^{80}$

$$
E_{\mathrm{CBS}}=\frac{(X+1)^{3} E_{X+1}-X^{3} E_{X}}{(X+1)^{3}-X^{3}}
$$

here $X$ denote the cardinal number of a basis set and $E_{X}$ is the corresponding energy. Dunning's correlation consistent basis sets are used throughout this work. ${ }^{81}$ The extrapolation scheme for noble gases is aug-cc-pVQZ $\rightarrow$ augcc-pV5Z; for all other systems the scheme used is aug-cc-pVTZ $\rightarrow$ aug-cc-pVQZ. The DFT energies and HF components of the wavefunction and RPA energies were obtained using the basis set with the largest cardinal number used for a given system and were not extrapolated.

Four different exchange-correlation functionals were used to generate the orbital input for RPA, namely $\mathrm{PBE}^{47}$ and its variant PBE0 including $25 \%$ of EXX, ${ }^{82}$ the meta-GGA SCAN ${ }^{48}$ and SCANO which is its hybrid variant with 
$25 \%$ of EXX. ${ }^{83}$ The label RPA $(X)$ corresponds to the RPA energy evaluated with orbitals and orbital energies of the DFT exchangecorrelation model $X$. $\operatorname{RPA}(X)+\mathrm{RSE}$ denotes RPA with the addition of the renormalized singles energy. ${ }^{44}$

\subsection{Noble gas dimers}

The vast majority of existing work on RPA for noncovalent systems is related to the interaction energies of molecular dimers ${ }^{30,31}$ or adsorption energies. ${ }^{33-35}$ As our conclusions regarding three-body systems are best understood in the context of two-body results, we briefly demonstrate the performance of RPA for the dimers of neon and argon. In particular, we are interested in the comparison of DFT vs. RPA, the differences between RPA energies computed with different orbital sets, and the effect of the singles correction.

Semilocal and DFT functionals lack longrange correlation and as a consequence the interaction energy decays too quickly in the tail region of both neon and argon. As expected, RPA, which correctly accounts for the dispersion energy, corrects for that deficiency for all orbital sets, see Figures 1 and 2.

At the equilibrium separation and in its vicinity, the DFT error becomes more difficult to predict. Two principal sources of error, the missing long-range dispersion and artificial binding due to the exchange energy, determine the total deviation. For neon, all DFT functionals except for PBE0 overbind, while for argon all DFT methods underbind.

The variation of the RPA results with the change of the orbital set is limited, but quantitatively important. As evident from Figures 1 and 2, RPA without RSE underestimates the curve depth around the equilibrium. The RSE correction always improves the energy upon bare RPA for argon. As expected, the RSE correction is smaller for the hybrid functionals. For $\mathrm{Ne}_{2}$, RPA+RSE using hybrid DFT orbitals is closer to the reference than for the pure DFT input. In contrast, using pure DFT gives better results for $\mathrm{Ar}_{2}$.

It is useful to compare the electron density predicted by DFT schemes with highly accurate density to identify the sources of errors and see the effect of the singles corrections. To this end we plot the difference between the density of the equilibrium Ne dimer at $R=3.1 \AA$ and the sum of isolated atom densities for each of the four approximate functionals, as well as for $\mathrm{HF}$ and for the coupled cluster (CC) schemes in Figure 3. The singles correction corresponds to the density obtained with a single $\mathrm{HF}$ iteration starting from the converged DFT self-consistent field. In the density difference plot, both HF and CC give charge depletion in the midpoint between the atoms while all the DFT approximations used show charge accumulation. While this artifact of approximate DFT could be partly caused by inaccurate correlation functional, inaccurate description of exchange is a more likely cause. This is because the difference between the HF and CC curves around midpoint is much smaller than the difference between the HF data and result of any DFT functional. Moreover, the incorrect accumulation is somewhat reduced when going from pure functionals to hybrids, also pointing to incorrect description of exchange. Finally, the addition of the singles correction has the largest effect, it completely removes the artifact and the density difference becomes closer to that of HF or CC.

\subsection{The 3B-69 test set}

We now turn to discuss the RPA results for predicting three-body energies in the 3B-69 test set of Řezáč and coworkers. ${ }^{45}$ The test set includes trimers of molecules composed of main-group elements. The reference energies employed in this work are taken from Ref. 45. The dataset avoids some of statistical biases by including a mix of systems which interact with a varying amount of nonadditive dispersion.

First we consider the RPA variants without the RSE correction. The mean signed errors (MSE), mean unsigned errors (MUE), and root-mean-square errors (RMSE) for RPA run with PBE, PBE0, and SCAN0 inputs are summarized in Table 3. The three-body nonadditive contributions improve when going from 


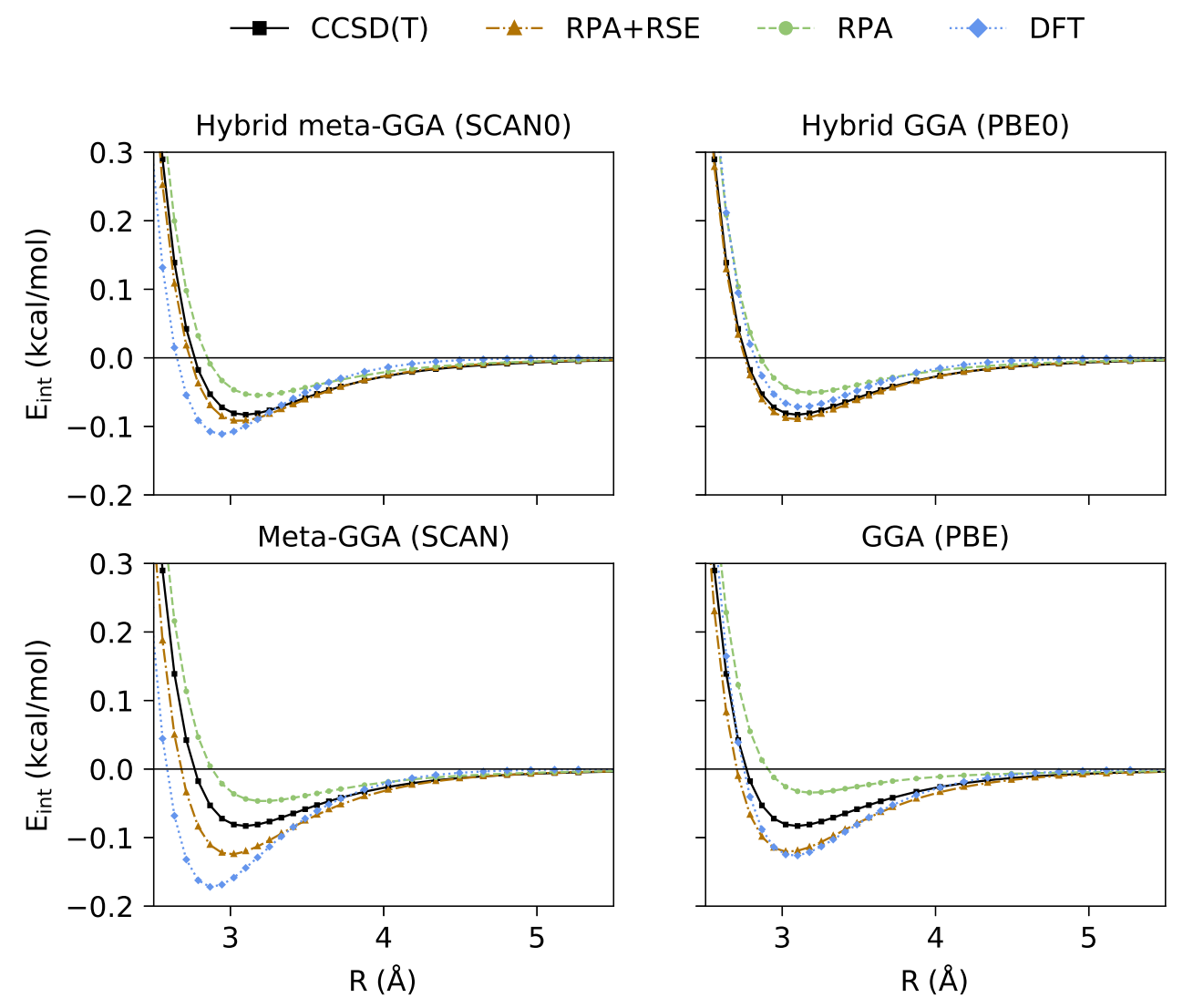

Figure 1: Neon dimer interaction energy computed using four sets of DFT orbitals.

the simplest to the most advanced exchangecorrelation model. Specifically, the RMSEs decrease from $0.054 \mathrm{kcal} / \mathrm{mol}$ for $\mathrm{RPA}(\mathrm{PBE})$, over $0.033 \mathrm{kcal} / \mathrm{mol}$ for $\mathrm{RPA}(\mathrm{PBE} 0)$, to $0.023 \mathrm{kcal} / \mathrm{mol}$ for RPA(SCAN0). For all aforementioned variants of RPA the MSE are negative, meaning that the three-body energies are too attractive compared to the reference, see also Figure 4.

Without the presence of the RSE correction, we observe stark differences in RPA's performance across the low, medium, and high dispersion subsets of the 3B-69 dataset. For $\mathrm{RPA}(\mathrm{PBE} 0)$ and $\mathrm{RPA}(\mathrm{SCAN} 0)$, the average errors increase with the fraction of the dispersion energy component (Table 4). While the above observation may look trivial for wavefunction based methods, it is not obvious for DFT based schemes, including RPA. As an example, the performance of RPA(PBE) cannot be rationalized in a simple way. Due to the sources of error inherited from the PBE orbitals, e.g., in the exchange and polarization nonadditivities, the error distribution of $\mathrm{RPA}(\mathrm{PBE})$ is much more uniform across the subsets of 3B-69 compared to the other RPA variants.

We now turn to the results obtained for RPA with the RSE correction added. While RSE improved the accuracy of the RPA interaction energies for noble gas dimers regardless of the orbital set, this is no longer the case for the 3B-69 dataset. RPA(SCAN0)+RSE is nearly always worse than RPA(SCAN0). This reduced accuracy occurs for all the subsets, irrespective of the importance of dispersion, see Table 4 . In this case, the RSE correction might overcorrect the errors in the three-body energies and terms beyond RSE are probably required to improve the accuracy of RPA(SCAN0). For $\mathrm{RPA}(\mathrm{PBE} 0)$, the statistical errors decrease by 10 to $20 \%$ upon the addition of RSE. Here the largest improvement occurs in the high and medium dispersion subsets (Table 3). For the low dispersion subset, the errors increase for large negative three body energies and decrease for the positive ones. Typically, these 

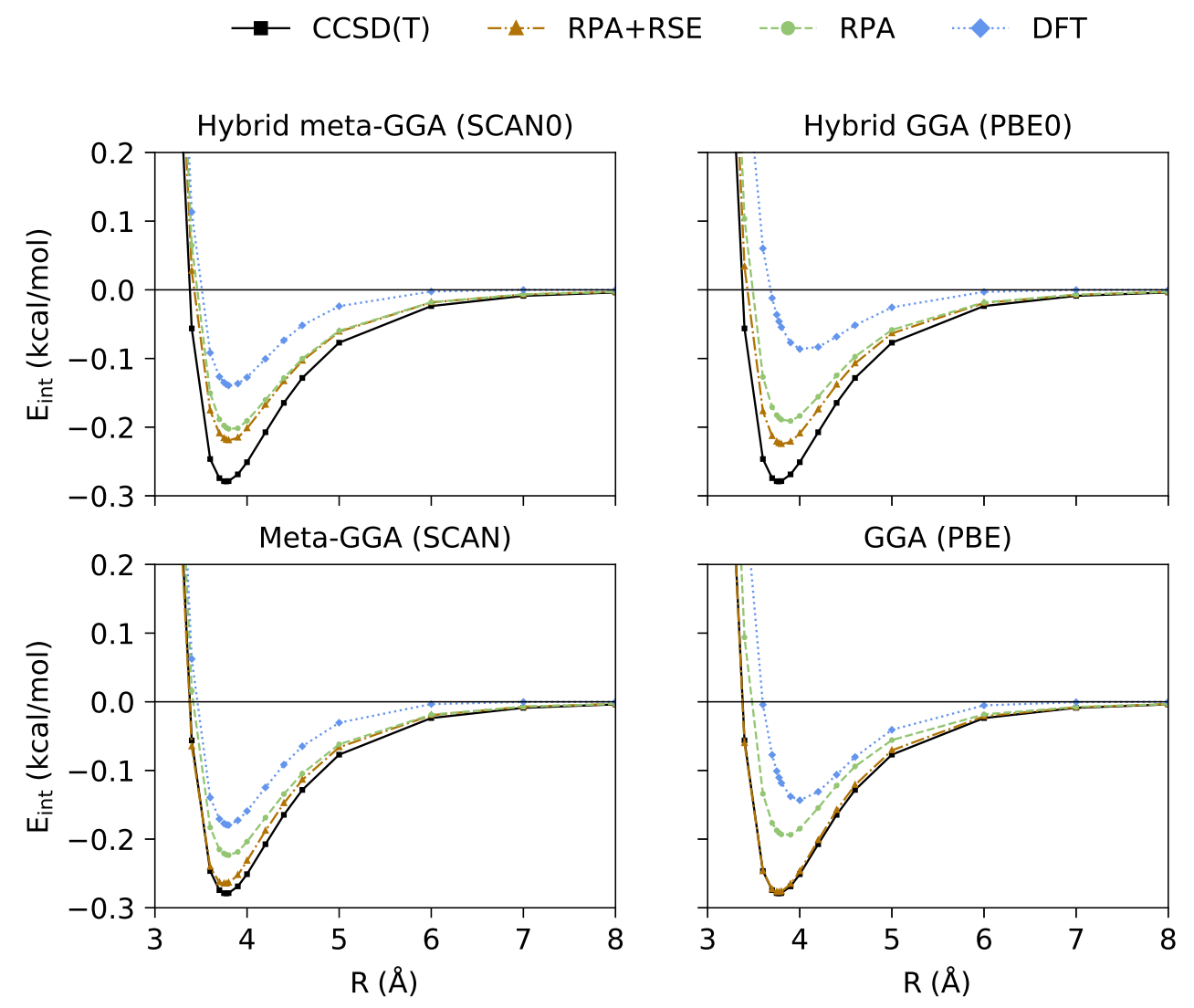

Figure 2: Argon dimer interaction energy computed using four sets of DFT orbitals. The reference coupled-cluster curve is taken from Ref. 84 .

correspond to systems with cooperative hydrogen bonds in the former case, and a hydrogen bonded dimer and a spectator molecule not taking part in the hydrogen bonding in the latter case. For RPA(PBE), we observe the same behavior of the RSE correction as for $\mathrm{RPA}(\mathrm{PBE} 0)$, only the reduction of errors brought by RSE is larger.

To establish the cost to accuracy ratio for RPA, we compare it to traditional postHF approaches. The worst RPA variant, $\mathrm{RPA}(\mathrm{PBE})$ is comparable in performance to MP2. The best performing RPA variants, that is, $\mathrm{RPA}(\mathrm{SCAN0})$ and $\mathrm{RPA}(\mathrm{PBE} 0)+\mathrm{RSE}$, are more accurate than MP2 and even better than MP3. This is a remarkable result considering the fact that MP3 scales with the sixth power of the system size, while the computational cost of RPA increases only with the third power. In fact, RPA(SCAN0) leads to an overall MUE of $0.018 \mathrm{kcal} / \mathrm{mol}$, this is only $30 \%$ larger than the MUE of $0.014 \mathrm{kcal} / \mathrm{mol}$ found for the MP2.5 approach and for the CCSD scheme. ${ }^{45}$

We now attempt to gain additional insight into the performance of the best performing variant, $\mathrm{RPA}(\mathrm{SCAN0})$. We identify the systems for which RPA(SCAN0) exhibits exceptionally large and exceptionally small errors, i.e., the challenging and easy subsets according to the relative deviations of $E_{\text {int }}[3,3]$ from the reference. The first nine systems with the errors above $50 \%$ are shown in Table 5 as the challenging subset; the nine trimers with errors below $0.5 \%$ are shown as the easy subset. The challenging systems, e.g., the trimers of benzene and uracil, are characterized by two features: (i) the share of the third-order contributions to $E_{\text {int }}[3,3]$ is large, that is,

$$
\left|\frac{E_{\text {int }}[3,3](\mathrm{MP} 3)-E_{\text {int }}[3,3](\mathrm{MP} 2)}{E_{\text {int }}[3,3](\mathrm{CCSD}(\mathrm{T}))}\right|>1
$$

and (ii) the magnitude of the total nonadditive interaction energy is small. In those cases the RPA's errors are more apparent than in 

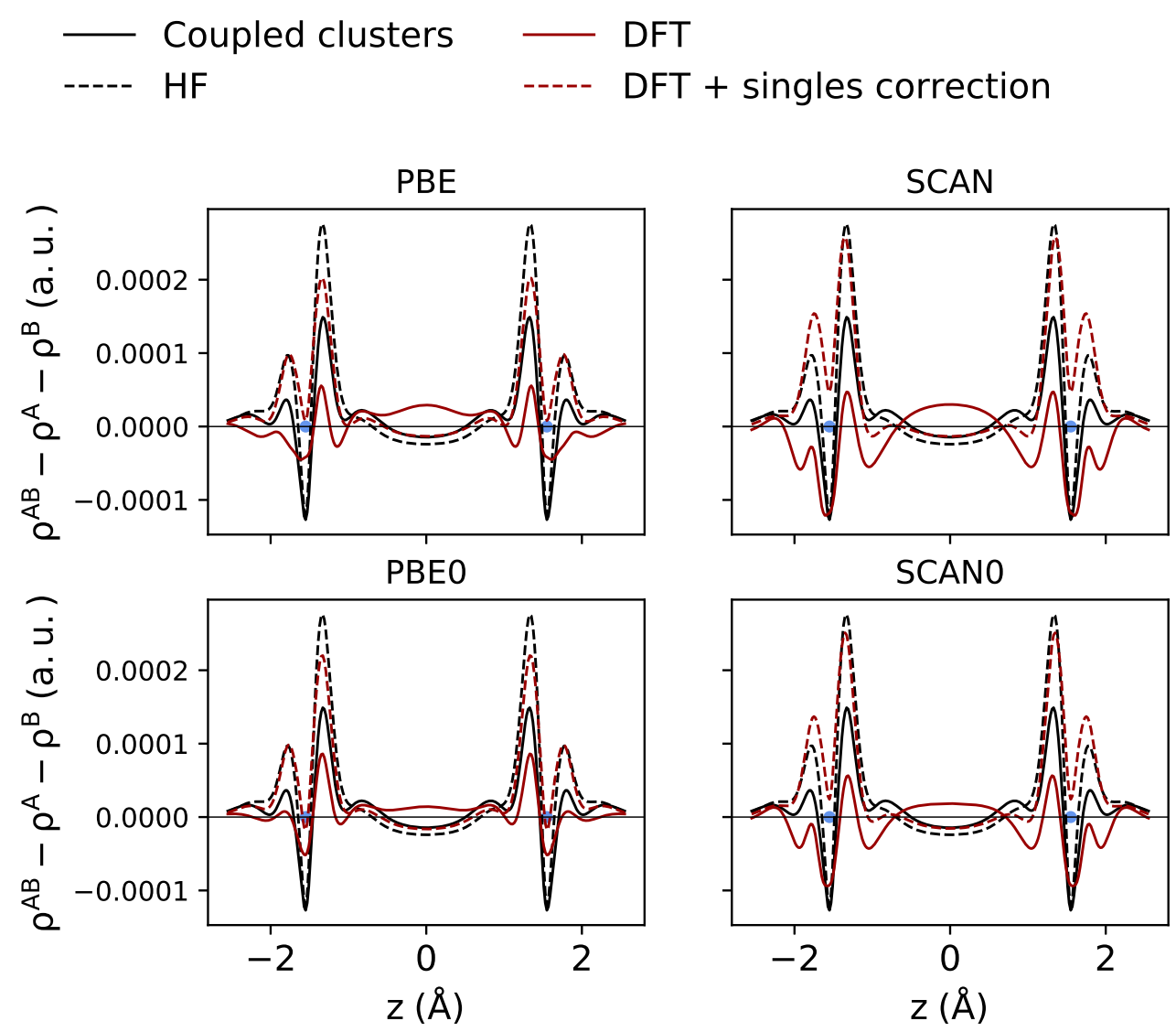

Figure 3: Change in DFT and coupled-cluster electron densities induced by the interaction in the Ne dimer at $R=3.1 \AA$. The atoms are placed at $z= \pm 1.55 \AA$. The drawn density resides at the interval $A B$ between endpoints $A=(0.125,0,-2.55) \AA$ and $B=(0.125,0,+2.55) \AA$, i.e., on the line placed $0.125 \AA$ above the bond axis. The CC3-level coupled-clusters density is generated using the software described in Refs. 85 and 86. Both coupled-cluster and DFT calculations employ the aug-cc-pCVQZ basis.

induction-dominated systems, where the total nonadditive interaction is generally stronger.

Most of the easy systems, e.g., trimers of water and acetic acid, are polar. The share of third-order Møller-Plesset contributions in $E_{\text {int }}[3,3]$ is small, and the magnitude of $E_{\text {int }}[3,3]$ is large. Alternatively, the threebody dispersion contribution in those systems is small compared to the total interaction (Table 5).

Let us briefly discuss the relation between the errors of the DFT functionals and the errors of RPA used to perform the subsequent calculations. First, one can notice that the results of PBE0 are, in terms of statistics, better than the results of RPA(PBE), see Table 4. Even more, for the high dispersion subset, the MSE and MUE of PBE0 are on par with those of RPA(SCAN0), the best RPA scheme tested here. Specifically, the error of PBE0 for the last challenging trimer, cyclobutylfuran $23 \mathrm{a}$, is only $-0.005 \mathrm{kcal} / \mathrm{mol}$ while RPA(PBE0) differs by $-0.061 \mathrm{kcal} / \mathrm{mol}$ from the reference. However, the good performance for PBE0 is a result of cancellation of errors between lack of long range correlation and spurious exchange binding. Moreover, the $(\mathrm{T})$ terms, not accounted for in RPA, and amounting to $0.039 \mathrm{kcal} / \mathrm{mol}$ for the cyclobutylfuran 23a trimer, could represent a part of the RPA error in this and similar cases.

\subsection{Noble gas trimers}

The statistical measures of the RPA results indicate that the most challenging trimers for 
Table 3: Average errors (kcal/mol) for the 3B-69 set of trimers. ${ }^{45}$ Data for the Møller-Plesset perturbation theory approximations are taken from Ref. 45.

\begin{tabular}{lrrr}
\hline Method & MSE & MUE & RMSE \\
\hline RPA(SCAN0) & -0.017 & 0.018 & 0.023 \\
RPA(SCAN0)+RSE & -0.027 & 0.028 & 0.034 \\
RPA(PBE0) & -0.026 & 0.026 & 0.033 \\
RPA(PBE0)+RSE & -0.020 & 0.023 & 0.029 \\
RPA(PBE) & -0.041 & 0.044 & 0.054 \\
RPA(PBE)+RSE & -0.013 & 0.026 & 0.038 \\
MP2 & -0.039 & 0.045 & 0.059 \\
MP3 & 0.022 & 0.026 & 0.035 \\
MP2.5 & -0.009 & 0.014 & 0.019 \\
SCAN0 & -0.054 & 0.065 & 0.081 \\
PBE0 & 0.017 & 0.039 & 0.053 \\
PBE & 0.068 & 0.093 & 0.116 \\
\hline
\end{tabular}

RPA(DFT) and RPA(DFT)+RSE are weakly interacting systems with a high share of the dispersion energy. To better understand the source of RPA's errors for those systems, as well as the sensitivity to the input orbital set, we turn to a case study of noble gas trimers.

The systems we consider are trimers of neon and argon in different configurations. Specifically, we use two isosceles triangle configurations with angles of 45 and 63.4 degrees and a linear configuration. See Figure 5 for the definition of the geometric parameters. The linear configurations correspond to a negative asymptote of the three-body dispersion energy; the remaining configurations correspond to a positive asymptote. The geometries and tabulated numerical data are included in the Supporting Information.

The nonadditive interaction energy curves are shown in Figures 6 and 7 for neon and argon, respectively. One can see that the performance of RPA(DFT) clearly depends on the input orbitals. The RPA(PBE) scheme, with and without RSE, clearly performs the worst with the interaction energy curve visibly further from the reference compared to the remaining methods. RPA based on PBE0 is of poorer quality than the approaches with the SCAN and SCAN0 orbitals, but it achieves a similar accuracy once the RSE correction is included. For RPA(PBE) and RPA(PBE0), the
RSE correction is necessary to reproduce the local maxima on the energy curves. In contrast, RPA(SCAN) and RPA(SCAN0) are qualitatively correct even without RSE. Quantitatively, RPA(PBE0)+RSE and the approaches based on the SCAN and SCAN0 achieve a similar level of accuracy.

We show the data obtained with SCAN0 for comparison in Figures 6 and 7. It performs rather well for the triangle configurations where there is a density overlap of the three atoms. However, it lacks the attractive three-body dispersion interaction needed to describe the binding curve of the trimer.

To rationalize the differences between various orbital sets, we note that the long distance decay of the electron density is controlled by the ionization potential (IP), which equals, by Janak's theorem, negative the HOMO eigenvalue. As seen in Table 6, the IPs at the DFT level approach the experimental values from below. Otherwise stated, all DFT methods yield electron densities which are too diffuse, and significantly so because the errors in the IPs are on the order of tens of percent.

The ordering of methods in terms of increasing IPs is $\mathrm{PBE}<\mathrm{SCAN}<\mathrm{PBE} 0<\mathrm{SCAN0}$. That sequence correlates with the magnitude of the RPA nonadditive interaction energy components (Figure 8). Specifically, the magnitudes of the EXX energy, singles correction, 
Table 4: Average errors (kcal/mol) for the low, medium, and high dispersion subsets of the 3B69 set of trimers as defined by Řezáč and co-workers. ${ }^{45}$ Data for the Møller-Plesset perturbation theory approximations are taken from Ref. 45 .

\begin{tabular}{lrlrrrrrrr}
\hline & \multicolumn{3}{c}{ Low dispersion } & \multicolumn{3}{c}{ Medium dispersion } & \multicolumn{3}{c}{ High dispersion } \\
Method & MSE & MUE & RMSE & MSE & MUE & RMSE & MSE & MUE & RMSE \\
\hline RPA(SCAN0) & -0.013 & 0.014 & 0.019 & -0.019 & 0.019 & 0.024 & -0.022 & 0.023 & 0.026 \\
RPA(SCAN0)+RSE & -0.020 & 0.021 & 0.027 & -0.029 & 0.029 & 0.033 & -0.035 & 0.036 & 0.041 \\
RPA(PBE0) & -0.019 & 0.020 & 0.028 & -0.027 & 0.027 & 0.031 & -0.034 & 0.034 & 0.038 \\
RPA(PBE0)+RSE & -0.018 & 0.023 & 0.031 & -0.019 & 0.020 & 0.024 & -0.023 & 0.026 & 0.029 \\
RPA(PBE) & -0.039 & 0.044 & 0.057 & -0.036 & 0.040 & 0.049 & -0.049 & 0.049 & 0.055 \\
RPA(PBE)+RSE & -0.025 & 0.041 & 0.054 & -0.005 & 0.016 & 0.022 & -0.006 & 0.018 & 0.021 \\
MP2 & -0.015 & 0.027 & 0.039 & -0.044 & 0.048 & 0.061 & -0.064 & 0.066 & 0.074 \\
MP3 & 0.012 & 0.021 & 0.026 & 0.018 & 0.022 & 0.031 & 0.038 & 0.038 & 0.046 \\
MP2.5 & -0.002 & 0.012 & 0.016 & -0.013 & 0.016 & 0.022 & -0.013 & 0.015 & 0.020 \\
SCAN0 & -0.028 & 0.052 & 0.069 & -0.061 & 0.061 & 0.074 & -0.082 & 0.085 & 0.099 \\
PBE0 & 0.000 & 0.051 & 0.064 & 0.035 & 0.039 & 0.052 & 0.022 & 0.025 & 0.033 \\
PBE & 0.021 & 0.084 & 0.112 & 0.097 & 0.098 & 0.121 & 0.100 & 0.100 & 0.112 \\
\hline
\end{tabular}

and RPA correlation energy form the sequence $\mathrm{PBE}<\mathrm{PBE} 0<\mathrm{SCAN}<\mathrm{SCAN0}$. Interestingly, the SCAN and PBE0 are reversed with respected to the order given by the IPs. We have computed the atomic density and found that, for the distance of interest, the atomic densities of PBE0 and SCAN are almost identical. Thus, the addition of Hartree-Fock exchange reduces the errors for both PBE an SCAN and using SCAN instead of PBE reduces the errors related to too delocalized states.

We now argue that, for the considered systems, the decay rate of the RPA energy components is an indication of the quality of the orbitals provided to the RPA energy formula. Because the EXX part of the RPA energy is based on the orbitals obtained with a hybrid or a semilocal DFT model, we expect it to account only for the physical terms that depend on the density overlap. Here, that would be the exchange nonadditivity and the intramonomer correlation corrections to it. ${ }^{89,90}$ By the above reasoning, the decay rate of the EXX components should be akin to that of HF and MP2, which respectively describe the abovementioned terms. However, we observe that the magnitude of the EXX energy is, for all tested orbital sets, significantly larger than that of HF and MP2 at long range and has a sign opposite to the
MP2 energy (Figure 8). Therefore, the large magnitude of EXX seen here is an artifact of approximate DFT functionals.

The excessive EXX term is partially cancelled by the RPA correlation, which has to be much larger in magnitude than the accurate three-body dispersion for the compensation to occur. The singles correction partakes in the cancellation of EXX, but does not remove the artifact entirely. A reliance on the cancellation of unphysical contributions appears to deteriorate the results for $\mathrm{RPA}(\mathrm{PBE})$ and $\mathrm{RPA}(\mathrm{PBE})+\mathrm{RSE}$, as those are the methods with the largest amount of cancellation between the different terms and also the worst performers for the noble gas trimers.

A step beyond RPA(DFT)+RSE would be an application of the GW singles correction described in Ref. 44 and applied, e.g., for the phase diagram of ice ${ }^{39}$ and for the binding energy curve of water on graphene. ${ }^{35}$ While due to technical reasons it is currently not possible to run large scale computations of GWSE for molecules using VASP, we have computed GWSE for a single neon trimer at $\theta=45^{\circ}$ and $R=3.0 \AA$ to probe its effect for the systems considered in this work (see Table 7). For $\mathrm{RPA}(\mathrm{SCAN})$, the difference between GWSE and RSE corrections is on the same order of 
Table 5: Nonadditive energies for the challenging and easy subsets of the 3B-69 dataset. The subsets are specified according to the relative error of the RPA(SCAN0) nonadditive energy (explained in the main text). Energies are in $\mathrm{kcal} / \mathrm{mol}$. The reference, MP2, and MP3 energies are taken from Ref. 45 . Here, $E_{\text {disp }}$ denotes the uncoupled ${ }^{87}$ three-body dispersion energy extrapolated using the aug-cc-pVTZ and aug-cc-pVQZ basis sets. RPA and RPA+RSE employ the SCAN0 orbitals.

\begin{tabular}{lrrrrrr}
\hline System & Ref. & $E_{\text {disp }}$ & MP2 & MP3 & RPA & RPA+RSE \\
\hline \multicolumn{7}{c}{ Challenging subset } \\
\hline CH $\left._{3} \mathrm{OH}\right)_{2}$ - ethyne (3c) & 0.023 & 0.037 & -0.003 & 0.089 & 0.008 & -0.002 \\
pyrazole (12b) & 0.067 & 0.129 & 0.010 & 0.114 & 0.026 & 0.005 \\
triazine (13a) & -0.005 & 0.008 & 0.013 & 0.002 & -0.011 & -0.010 \\
succinic anhydride (18b) & -0.001 & -0.003 & 0.003 & 0.001 & 0.007 & 0.004 \\
benzene (19a) & 0.048 & 0.204 & -0.054 & 0.126 & 0.021 & -0.012 \\
benzene (19c) & -0.027 & 0.085 & -0.061 & 0.016 & -0.044 & -0.053 \\
p-benzoquinone (21b) & 0.004 & 0.058 & -0.038 & 0.006 & -0.012 & -0.028 \\
uracil (22a) & -0.004 & 0.068 & -0.033 & 0.006 & -0.010 & 0.005 \\
cyclobutylfuran (23a) & 0.081 & 0.274 & -0.049 & 0.186 & 0.031 & -0.009 \\
\hline & & Easy subset & & & \\
\hline water (1c) & -2.416 & 0.068 & -2.472 & -2.404 & -2.411 & -2.461 \\
acetonitrile (4c) & -0.166 & -0.005 & -0.155 & -0.132 & -0.165 & -0.168 \\
nitromethane (5c) & 0.220 & -0.007 & 0.216 & 0.229 & 0.220 & 0.217 \\
acetic acid (6a) & 0.542 & 0.031 & 0.523 & 0.558 & 0.541 & 0.544 \\
oxalic acid (7b) & -1.198 & 0.012 & -1.199 & -1.170 & -1.201 & -1.228 \\
acetamide (9c) & -0.860 & -0.003 & -0.850 & -0.869 & -0.858 & -0.863 \\
imidazole (10c) & -1.636 & -0.013 & -1.631 & -1.608 & -1.628 & -1.665 \\
maleic acid (20b) & -1.449 & -0.004 & -1.428 & -1.419 & -1.451 & -1.496 \\
p-benzoquinone (21c) & 0.096 & 0.090 & 0.039 & 0.126 & 0.096 & 0.070 \\
\hline
\end{tabular}

Table 6: Ionization potentials (eV) for isolated noble gas atoms, computed with Koopmans's theorem. The experimental IPs are taken from the NIST Atomic Spectra Database. ${ }^{88}$

\begin{tabular}{lrr}
\hline Method & $\mathrm{Ne}$ & $\mathrm{Ar}$ \\
\hline SCAN0 & 16.5 & 12.3 \\
PBE0 & 16.0 & 12.0 \\
SCAN & 14.0 & 10.7 \\
PBE & 13.3 & 10.3 \\
exp. & 21.6 & 15.8 \\
\hline
\end{tabular}

Table 7: Effect of the RSE and GW singles correction on the nonadditive interaction energy $(\mathrm{kcal} / \mathrm{mol})$ of $\mathrm{Ne}_{3}$ at angle $45^{\circ}$ and $R=3.0 \AA$.

\begin{tabular}{lr}
\hline Method & $E_{\text {int }}[3,3]$ \\
\hline CCSD $(T)$ & 0.0040 \\
RPA(SCAN) & -0.0057 \\
RPA(SCAN)+RSE & 0.0213 \\
RPA(SCAN)+GWSE & 0.0163 \\
RPA(SCAN0) & -0.0009 \\
RPA(SCAN0)+RSE & 0.0108 \\
\hline
\end{tabular}

magnitude as the reference interaction energy at the considered distance and comparable to the effect of changing the orbital set from SCAN to SCAN0. A futher investigation of the GWSE correction remains a subject of our future work. 

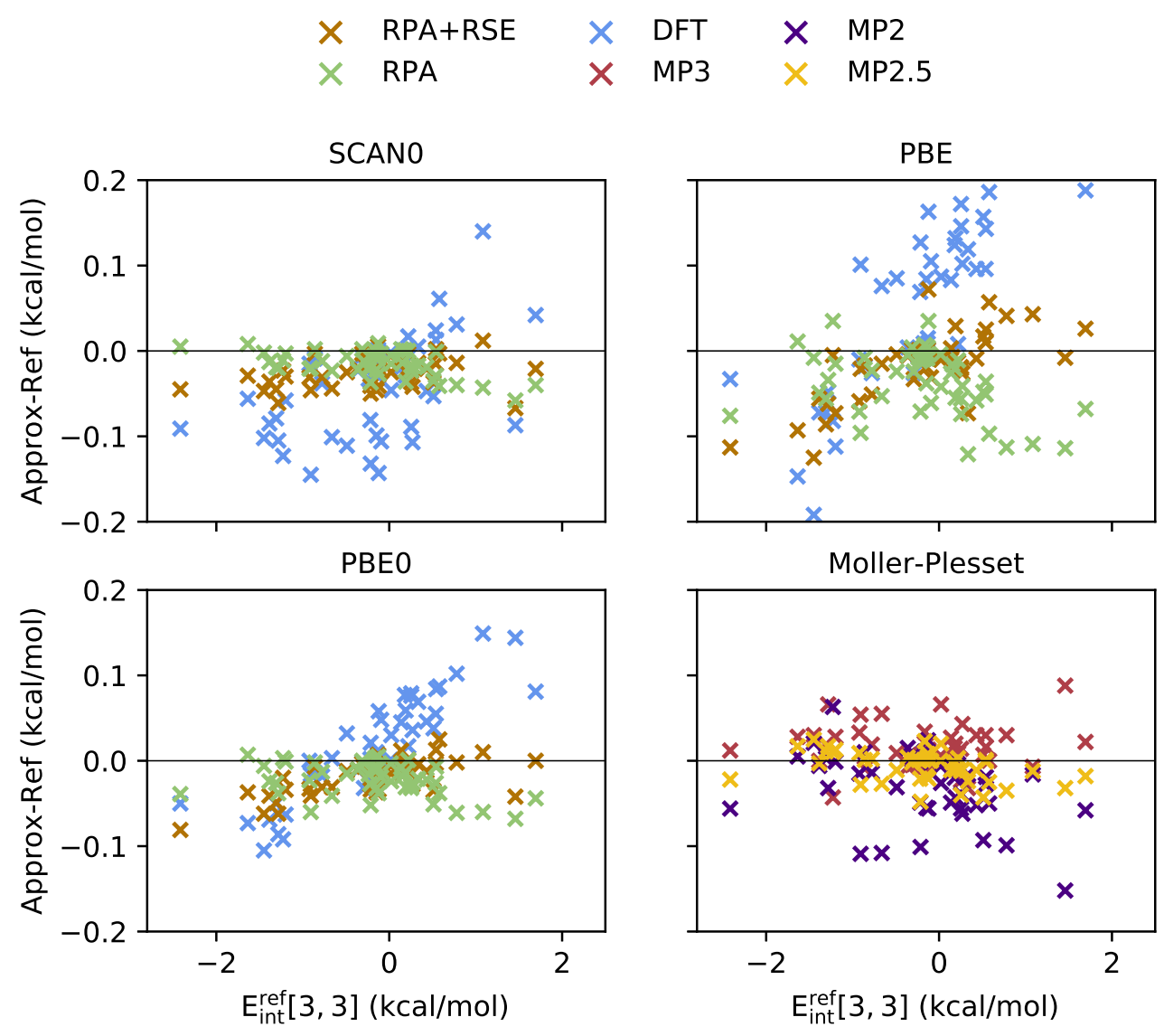

Figure 4: Signed errors of approximate methods vs the reference nonadditive interaction energy (kcal/mol) on the 3B-69 test set. ${ }^{45}$

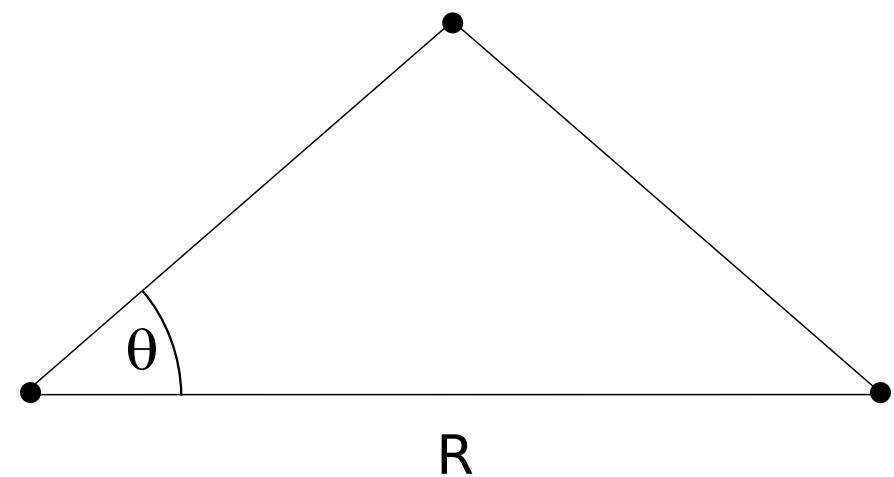

Figure 5: Parameters specifying the isosceles triangle configurations of $\mathrm{Ne}_{3}$ and $\mathrm{Ar}_{3}$.

\section{Conclusions}

We have examined numerical and theoretical aspects of applying RPA for many-body noncovalent systems of atoms and molecules. We introduced a cubic scaling algorithm for molecular RPA which achieves high and controllable numerical precision. Unlike prior efficient RPA implementations, the cubic scaling does not assume the sparsity of the effective density matrices, or Green's functions. It employs a systematically improvable Cholesky basis instead of the usual auxiliary basis sets for the decomposition of the Coulomb matrix. Those features make the algorithm fit for accumulating subtle $n$-body contributions in clusters of interacting molecules.

Regarding the accuracy of nonadditive interaction energies at the RPA level, the choice of orbitals affects RPA quantitatively and, in some cases, qualitatively. To assess RPA's dependence on the Kohn-Sham state, we tested GGA and meta-GGA exchange-correlation models: PBE, PBE0, SCAN, and SCANO. In addition, we tested the singles correction, RSE, which effectively changes the electron density, affects the Hartree-Fock part of the RPA energy, but does not affect the RPA correlation contribution. 

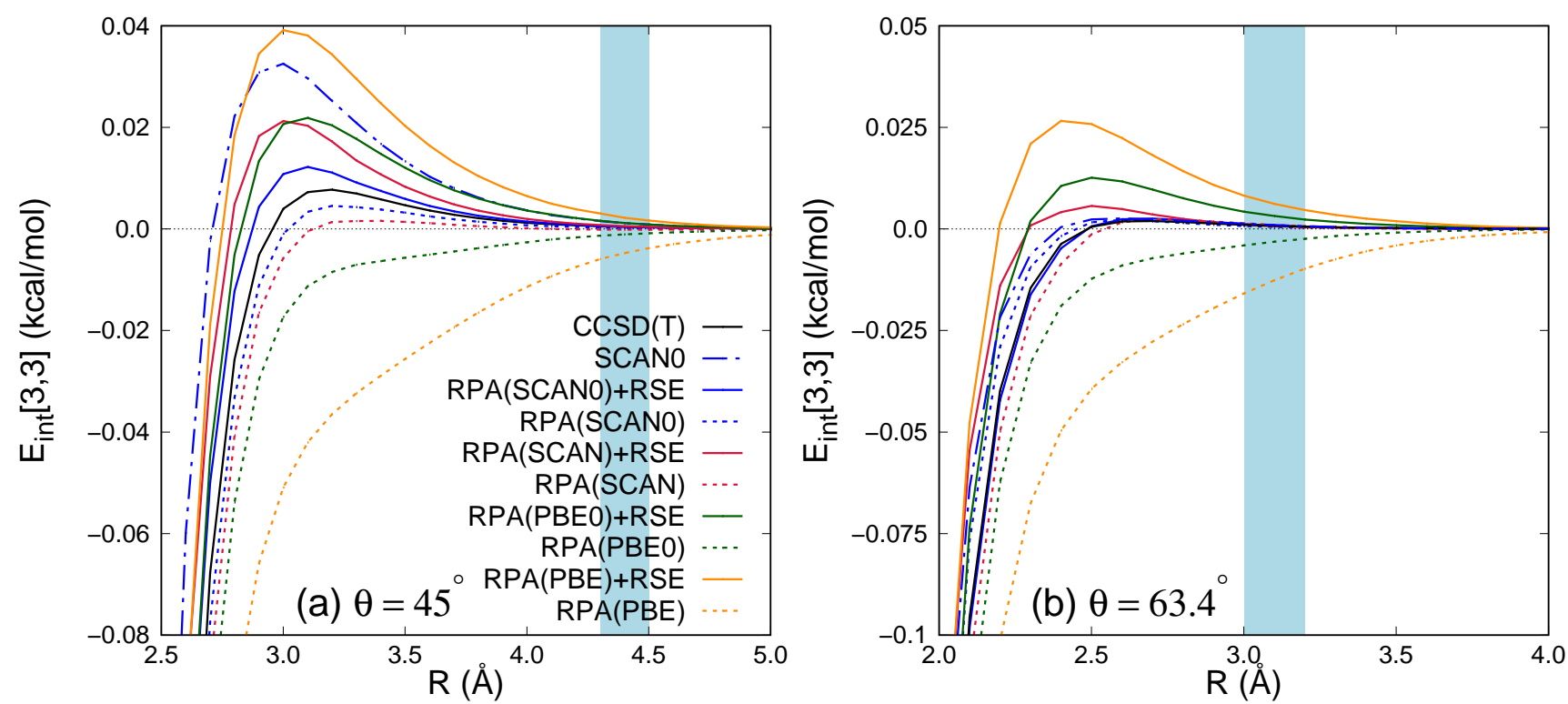

Figure 6: Nonadditive three-body interaction energy of neon trimers. The blue background denotes configurations where the closest pair of atoms is within $\pm 0.1 \AA$ of the equilibrium dimer separation.

Our statistical data on the 3B-69 set of trimers demonstrate that the best RPA variants are based on SCAN0 hybrid meta-GGA (applied without RSE) and PBE0 hybrid GGA (applied in combination with RSE). The RPA methods achieve a much better accuracy than their base DFT functionals. Compared to wavefunction methods, the accuracy of RPA(SCAN0) and RPA(PBE0)+RSE is between the MP3 and CCSD approaches, which have orders of magnitude larger requirements of storage and compute time. For solid state calculations hybrid functionals incur additional computational cost. Our data for noble gas trimers suggest that when hybrid DFT calculations are not feasible, SCAN is currently the best choice of a pure DFT model. Following the standard practice, that is using RPA with PBE orbitals, gives the worst predictions over the entire 3B-69 dataset. The results for noble gas trimers suggest that the reason behind the poor performance of RPA(PBE) and $\mathrm{RPA}(\mathrm{PBE})+\mathrm{RSE}$ is that the Hartree-Fock part of the RPA interaction energy in those cases decays at an artificially slow rate as a function of the intermonomer distance.

Out of two major advantages that RPA has over semilocal DFT, that is, the account of the dispersion energy and the compatibility with exact exchange, the latter appears to be especially important as it eliminates the artificial exchange overlap interactions already reported in the DFT literature. ${ }^{19}$ As a result we observe a near-benchmark accuracy of our best RPA variants for the low-dispersion subset of the 3B69 dataset.

Overall, we find that RPA is, in terms of computational cost and accuracy, a well balanced scheme for predicting many-body energies of systems bound by noncovalent interactions. Its accuracy to cost ratio makes it a method preferable to both hybrid semilocal DFT and simple wave function approaches, e.g., MP2 and MP3. We have identified that the DFT errors of the base functional visibly transfer to the RPA results, which implies that a further improvement of the RPA methodology is still possible by devising better schemes for generating the orbital input.

Acknowledgement This work was supported by the European Research Council (ERC) under the European Union's Horizon 2020 research and innovation program (grant agreement No 759721). We are grateful for the computational resources provided by the IT4Innovations National Supercomputing Center (LM2015070), CESNET (LM2015042), and 

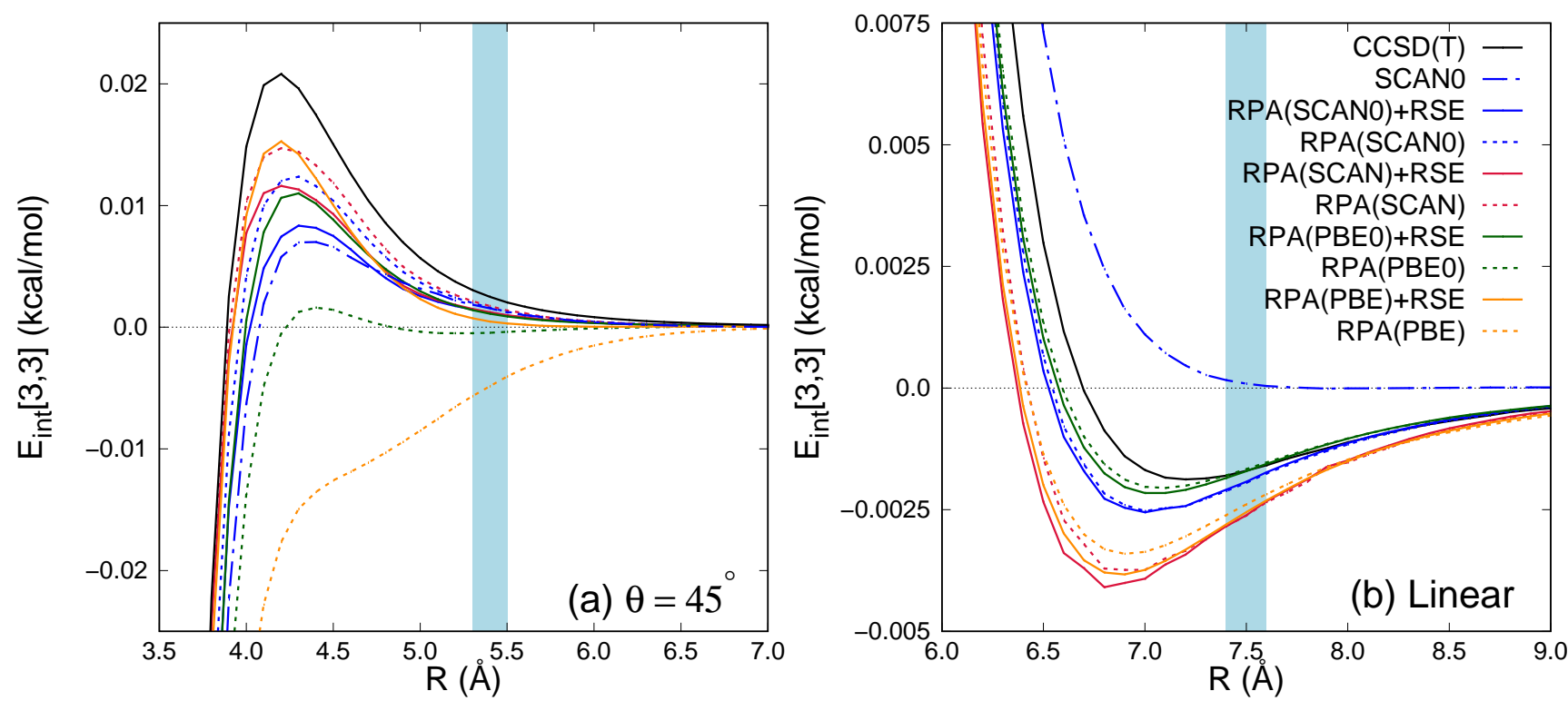

Figure 7: Nonadditive three-body interaction energy of argon trimers. The blue background denotes configurations where the closest pair of atoms is within $\pm 0.1 \AA$ of the equilibrium dimer separation.

CERIT-SC (LM2015085) funded within the programme "Projects of Large Research, Development, and Innovations Infrastructures" of the Ministry of Education, Youth, and Sports. This research was supported in part by PLGrid Infrastructure. We thank David P. Tew for help with obtaining initial results. We thank Aleksandra Tucholska for giving us access to her coupled-cluster program for generating electronic densities.

\section{Supporting Information Avail- able}

The following files are available free of charge. Spreadsheets with raw numerical data and computational details, geometries in a form of xyz files. The supporting information is available free of charge via the Internet at https://pubs.acs.org/doi/10.1021/acs.jctc.9b00979

\section{References}

(1) Price, S. L. Computer crystal energy landscapes for understanding and predicting organic crystal structures and polymorphism. Acc. Chem. Res. 2009, 42, 117.
(2) Nyman, J.; Day, G. M. Static and lattice vibrational energy differences between polymorphs. CrystEngComm 2015, 17, 5154-5165.

(3) Reilly, A. M.; Cooper, R. I.; Adjiman, C. S.; Bhattacharya, S.; Boese, A. D.; Brandenburg, J. G.; Bygrave, P. J.; Bylsma, R.; Campbell, J. E.; Car, R.; Case, D. H.; Chadha, R.; Cole, J. C.; Cosburn, K.; Cuppen, H. M.; Curtis, F.; Day, G. M.; DiStasio Jr, R. A.; Dzyabchenko, A.; van Eijck, B. P.; Elking, D. M.; van den Ende, J. A.; Facelli, J. C.; Ferraro, M. B.; Fusti-Molnar, L.; Gatsiou, C.-A.; Gee, T. S.; de Gelder, R.; Ghiringhelli, L. M.; Goto, H.; Grimme, S.; Guo, R.; Hofmann, D. W. M.; Hoja, J.; Hylton, R. K.; Iuzzolino, L.; Jankiewicz, W.; de Jong, D. T.; Kendrick, J.; de Klerk, N. J. J.; Ko, H.-Y.; Kuleshova, L. N.; Li, X.; Lohani, S.; Leusen, F. J. J.; Lund, A. M.; Lv, J.; Ma, Y.; Marom, N.; Masunov, A. E.; McCabe, P.; McMahon, D. P.; Meekes, H.; Metz, M. P.; Misquitta, A. J.; Mohamed, S.; Monserrat, B.; Needs, R. J.; Neumann, M. A.; Nyman, J.; Obata, S.; Oberhofer, H.; Oganov, A. R.; Orendt, A. M.; Pagola, G. I.; Pantelides, C. C.; Pickard, C. J.; Podeszwa, R.; Price, L. S.; Price, S. L.; Pulido, A.; Read, M. G.; Reuter, K.; Schneider, E.; Schober, C.; Shields, G. P.; Singh, P.; Sugden, I. J.; Szalewicz, K.; Taylor, C. R.; Tkatchenko, A.; Tuckerman, M. E.; Vacarro, F.; Vasileiadis, M.; Vazquez-Mayagoitia, A.; Vogt, L.; Wang, Y.; Watson, R. E.; de Wijs, G. A.; Yang, J.; Zhu, Q.; Groom, C. R. Report on the sixth blind test of organic crystal structure prediction methods. Acta Crystallographica Section B 2016, 72, 439-459. 


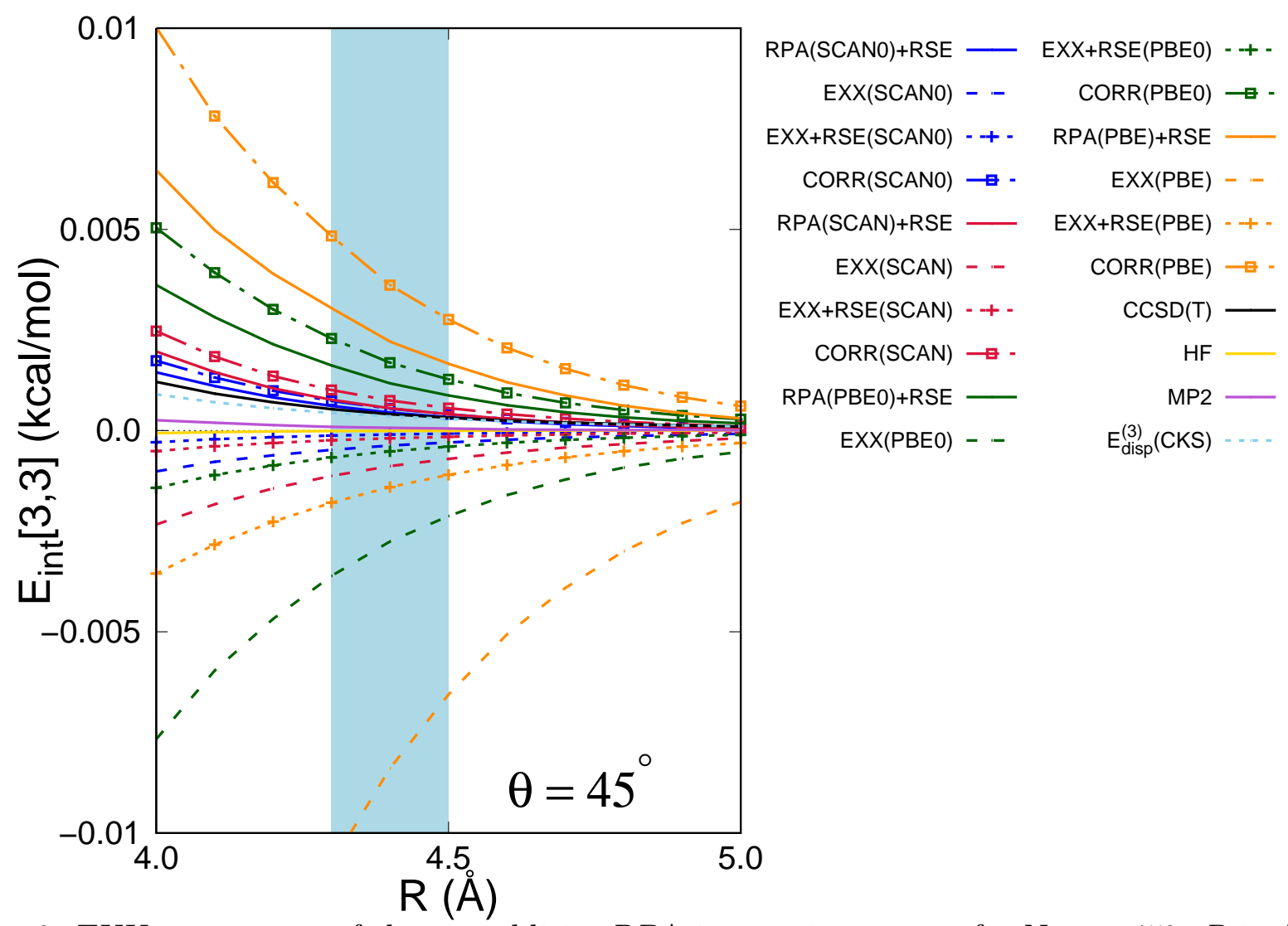

Figure 8: EXX component of the nonadditive RPA interaction energy for $\mathrm{Ne}_{3}$ at $45^{\circ} . R$ is the length of the isosceles triangle base. The blue background denotes configurations where the closest pair of atoms is within $\pm 0.1 \AA$ of the equilibrium dimer separation.

(4) Červinka, C.; Beran, G. J. Ab initio prediction of the polymorph phase diagram for crystalline methanol. Chemical science 2018, 9, 4622-4629.

(5) Addicoat, M.; Adjiman, C. S.; Arhangelskis, M.; Beran, G. J. O.; Bowskill, D.; Brandenburg, J. G.; Braun, D. E.; Burger, V.; Cole, J.; CruzCabeza, A. J.; Day, G. M.; Deringer, V. L.; Guo, R.; Hare, A.; Helfferich, J.; Hoja, J.; Iuzzolino, L.; Jobbins, S.; Marom, N.; McKay, D.; Mitchell, J. B. O.; Mohamed, S.; Neumann, M.; Nilsson Lill, S.; Nyman, J.; Oganov, A. R.; Piaggi, P.; Price, S. L.; Reutzel-Edens, S.; Rietveld, I.; Ruggiero, M.; Ryder, M. R.; Sastre, G.; Schön, J. C.; Taylor, C.; Tkatchenko, A.; Tsuzuki, S.; van den Ende, J.; Woodley, S. M.; Woollam, G.; Zhu, Q. Crystal structure evaluation: calculating relative stabilities and other criteria: general discussion. Faraday Discuss. 2018, 211, 325-381.

(6) Hoja, J.; Ko, H.-Y.; Neumann, M. A.; Car, R.; DiStasio, R. A.; Tkatchenko, A. Reliable and practical computational description of molecular crystal polymorphs. Sci. Adv. 2019, 5, eaau3338.

(7) Kennedy, M. R.; McDonald, A. R.; DePrince, A. E.; Marshall, M. S.; Podeszwa, R.; Sher- rill, C. D. Communication: Resolving the threebody contribution to the lattice energy of crystalline benzene: Benchmark results from coupledcluster theory. J. Chem. Phys. 2014, 140, 121104.

(8) Yang, J.; Hu, W.; Usvyat, D.; Matthews, D.; Schütz, M.; Chan, G. K.-L. Ab initio determination of the crystalline benzene lattice energy to sub-kilojoule/mole accuracy. Science 2014, 345, 640-643.

(9) Lotrich, V. F.; Szalewicz, K. Three-body contribution to binding energy of solid argon and analysis of crystal structure. Phys. Rev. Lett. 1997, 79, 1301.

(10) Góra, U.; Podeszwa, R.; Cencek, W.; Szalewicz, K. Interaction energies of large clusters from manybody expansion. J. Chem. Phys. 2011, 135, 224102.

(11) Gillan, M. J.; Alfè, D.; Bygrave, P. J.; Taylor, C. R.; Manby, F. R. Energy benchmarks for water clusters and ice structures from an embedded many-body expansion. J. Chem. Phys. 2013, $139,114101$. 
(12) Gillan, M. J.; Alfè, D.; Michaelides, A. Perspective: How good is DFT for water? J. Chem. Phys. 2016, 144, 130901.

(13) Grimme, S.; Hansen, A.; Brandenburg, J. G.; Bannwarth, C. Dispersion-corrected mean-field electronic structure methods. Chem. Rev. 2016, 116, $5105-5154$

(14) Vydrov, O.; Van Voorhis, T. Nonlocal van der Waals density functional: The simpler the better. J. Chem. Phys. 2010, 133, 244103.

(15) Lee, K.; Murray, E.; Kong, L.; Lundqvist, B.; Langreth, D. Higher-accuracy van der Waals density functional. Phys. Rev. B 2010, 82, 081101.

(16) Grimme, S.; Antony, J.; Ehrlich, S.; Krieg, H. A consistent and accurate ab initio parametrization of density functional dispersion correction (DFTD) for the 94 elements H-Pu. J. Chem. Phys. 2010, 132, 154104.

(17) Caldeweyher, E.; Bannwarth, C.; Grimme, S. Extension of the D3 dispersion coefficient model. $J$. Chem. Phys. 2017, 147, 034112.

(18) Ambrosetti, A.; Reilly, A. M.; DiStasio Jr, R. A.; Tkatchenko, A. Long-range correlation energy calculated from coupled atomic response functions. $J$. Chem. Phys. 2014, 140, 18A508.

(19) Gillan, M. Many-body exchange-overlap interactions in rare gases and water. J. Chem. Phys. 2014, 141, 224106.

(20) Hapka, M.; Rajchel, L.; Modrzejewski, M.; Schaeffer, R.; Chalasinski, G.; Szczesniak, M. M. The nature of three-body interactions in DFT: Exchange and polarization effects. J. Chem. Phys. 2017, $147,084106$.

(21) Jankiewicz, W.; Podeszwa, R.; Witek, H. A. Dispersion-corrected DFT struggles with predicting three-body interaction energies. J. Chem. Theory Comput. 2018, 14, 5079-5089.

(22) Lacks, D. J.; Gordon, R. G. Pair interactions of rare-gas atoms as a test of exchange-energydensity functionals in regions of large density gradients. Phys. Rev. A 1993, 47, 4681.

(23) Deible, M. J.; Tuguldur, O.; Jordan, K. D. Theoretical Study of the Binding Energy of a Methane Molecule in a $\left(\mathrm{H}_{2} \mathrm{O}\right)_{20}$ Dodecahedral Cage. $J$. Phys. Chem. B 2014, 118, 8257-8263.

(24) Lao, K. U.; Herbert, J. M. Accurate and efficient quantum chemistry calculations for noncovalent interactions in many-body systems: The XSAPT family of methods. J. Phys. Chem. A 2014, 119, 235-252.
(25) Huang, Y.; Beran, G. J. O. Reliable prediction of three-body intermolecular interactions using dispersion-corrected second-order Møller-Plesset perturbation theory. J. Chem. Phys. 2015, 143, 044113 .

(26) Lao, K. U.; Herbert, J. M. Atomic orbital implementation of extended symmetry-adapted perturbation theory (XSAPT) and benchmark calculations for large supramolecular complexes. $J$. Chem. Theory Comput. 2018, 14, 2955-2978.

(27) Carter-Fenk, K.; Lao, K. U.; Liu, K.-Y.; Herbert, J. M. Accurate and Efficient ab Initio Calculations for Supramolecular Complexes: Symmetry-Adapted Perturbation Theory with Many-Body Dispersion. J. Phys. Chem. Lett. 2019, 10, 2706-2714.

(28) Dobson, J. F. In Fundamentals of Time-Dependent Density Functional Theory; Marques, M. A., Maitra, N. T., Nogueira, F. M., Gross, E., Rubio, A., Eds.; Springer: Berlin, Heidelberg, 2012; pp 417-441.

(29) Dobson, J.; Gould, T. Calculation of dispersion energies. J. Phys.: Condens. Matter 2012, 24, 073201.

(30) Eshuis, H.; Furche, F. A Parameter-Free Density Functional That Works for Noncovalent Interactions. J. Phys. Chem. Lett. 2011, 2, 983-989.

(31) Bleiziffer, P.; Hesselmann, A.; Gorling, A. Efficient self-consistent treatment of electron correlation within the random phase approximation. $J$. Chem. Phys. 2013, 139, 084113.

(32) Ren, X.; Rinke, P.; Scuseria, G. E.; Scheffler, M. Renormalized second-order perturbation theory for the electron correlation energy: Concept, implementation, and benchmarks. Phys. Rev. B 2013, 88, 035120.

(33) Garrido Torres, J. A.; Ramberger, B.; Früchtl, H. A.; Schaub, R.; Kresse, G. Adsorption energies of benzene on close packed transition metal surfaces using the random phase approximation. Phys. Rev. Materials 2017, 1, 060803.

(34) Al-Hamdani, Y. S.; Rossi, M.; Alfè, D.; Tsatsoulis, T.; Ramberger, B.; Brandenburg, J. G.; Zen, A.; Kresse, G.; Grüneis, A.; Tkatchenko, A.; Michaelides, A. Properties of the water to boron nitride interaction: From zero to two dimensions with benchmark accuracy. J. Chem. Phys. 2017, $147,044710$. 
(35) Brandenburg, J. G.; Zen, A.; Fitzner, M.; Ramberger, B.; Kresse, G.; Tsatsoulis, T.; Gruneis, A.; Michaelides, A.; Alfe, D. Physisorption of water on graphene: Subchemical accuracy from many-body electronic structure methods. J. Phys. Chem. Lett. 2019, 10, 358-368.

(36) Lu, D.; Li, Y.; Rocca, D.; Galli, G. Ab initio Calculation of van der Waals Bonded Molecular Crystals. Phys. Rev. Lett. 2009, 102, 206411.

(37) Del Ben, M.; Hutter, J.; VandeVondele, J. Electron Correlation in the Condensed Phase from a Resolution of Identity Approach Based on the Gaussian and Plane Waves Scheme. J. Chem. Theory Comput. 2013, 9, 2654-2671.

(38) Klimeš, J. Lattice energies of molecular solids from the random phase approximation with singles corrections. J. Chem. Phys. 2016, 145, 094506.

(39) Zen, A.; Brandenburg, J. G.; Klimes, J.; Tkatchenko, A.; Alfe, D.; Michaelides, A. Fast and accurate quantum Monte Carlo for molecular crystals. Proc. Natl. Acad. Sci. U. S. A. 2018, 115, 1724-1729.

(40) Harl, J.; Kresse, G. Cohesive energy curves for noble gas solids calculated by adiabatic connection fluctuation-dissipation theory. Phys. Rev. B 2008, 77, 045136.

(41) Mittendorfer, F.; Garhofer, A.; Redinger, J.; Klimeš, J.; Harl, J.; Kresse, G. Graphene on $\mathrm{Ni}(111)$ : Strong interaction and weak adsorption. Phys. Rev. B 2011, 84, 201401.

(42) Olsen, T.; Thygesen, K. S. Random phase approximation applied to solids, molecules, and graphenemetal interfaces: From van der Waals to covalent bonding. Phys. Rev. B 2013, 87, 075111.

(43) Ren, X.; Tkatchenko, A.; Rinke, P.; Scheffler, M. Beyond the random-phase approximation for the electron correlation energy: The importance of single excitations. Phys. Rev. Lett. 2011, 106, 153003.

(44) Klimes, J.; Kaltak, M.; Maggio, E.; Kresse, G. Singles correlation energy contributions in solids. J. Chem. Phys. 2015, 143, 102816.

(45) Rezac, J.; Huang, Y.; Hobza, P.; Beran, G. J. Benchmark calculations of three-body intermolecular interactions and the performance of low-cost electronic structure methods. J. Chem. Theory Comput. 2015, 11, 3065.
(46) Hybertsen, M. S.; Louie, S. G. Ab initio static dielectric matrices from the density-functional approach. I. Formulation and application to semiconductors and insulators. Phys. Rev. B 1987, 35, 5585 .

(47) Perdew, J.; Burke, K.; Ernzerhof, M. Generalized Gradient Approximation Made Simple. Phys. Rev. Lett. 1996, 77, 3865-3868.

(48) Sun, J.; Ruzsinszky, A.; Perdew, J. P. Strongly Constrained and Appropriately Normed Semilocal Density Functional. Phys. Rev. Lett. 2015, 115, 036402 .

(49) Strubbe, D. A.; Lehtovaara, L.; Rubio, A.; Marques, M. A.; Louie, S. G. In Fundamentals of Time-Dependent Density Functional Theory; Marques, M. A., Maitra, N. T., Nogueira, F. M., Gross, E., Rubio, A., Eds.; Springer: Berlin, Heidelberg, 2012; pp 139-166.

(50) Graf, D.; Beuerle, M.; Ochsenfeld, C. Low-Scaling Self-Consistent Minimization of a Density Matrix Based Random Phase Approximation Method in the Atomic Orbital Space. J. Chem. Theory Comput. 2019, 15, 4468-4477.

(51) Gross, E. K. U.; Dobson, J. F.; Petersilka, M. In Density Functional Theory II: Relativistic and Time Dependent Extensions; Nalewajski, R. F., Ed.; Springer Berlin Heidelberg: Berlin, Heidelberg, 1996; pp 81-172.

(52) Furche, F.; Van Voorhis, T. Fluctuationdissipation theorem density-functional theory. $J$. Chem. Phys. 2005, 122, 164106.

(53) Kaltak, M.; Klimes, J.; Kresse, G. Low scaling algorithms for the random phase approximation: Imaginary time and laplace transformations. $J$. Chem. Theory Comput. 2014, 10, 2498.

(54) Eshuis, H.; Yarkony, J.; Furche, F. Fast computation of molecular random phase approximation correlation energies using resolution of the identity and imaginary frequency integration. J. Chem. Phys. 2010, 132, 234114.

(55) Ren, X.; Rinke, P.; Blum, V.; Wieferink, J.; Tkatchenko, A.; Sanfilippo, A.; Reuter, K.; Scheffler, M. Resolution-of-identity approach to Hartree-Fock, hybrid density functionals, RPA, MP2 and GW with numeric atom-centered orbital basis functions. New J. Phys. 2012, 14, 053020.

(56) Wilhelm, J.; Seewald, P.; Del Ben, M.; Hutter, J. Large-scale cubic-scaling random phase approximation correlation energy calculations using a Gaussian basis. J. Chem. Theory Comput. 2016, 12, 5851 . 
(57) Schurkus, H. F.; Ochsenfeld, C. Communication: An effective linear-scaling atomic-orbital reformulation of the random-phase approximation using a contracted double-Laplace transformation. $J$. Chem. Phys. 2016, $144,031101$.

(58) Luenser, A.; Schurkus, H. F.; Ochsenfeld, C. Vanishing-overhead linear-scaling random phase approximation by Cholesky decomposition and an attenuated Coulomb-metric. J. Chem. Theory Comput. 2017, 13, 1647.

(59) Aquilante, F.; Boman, L.; Bostrom, J.; Koch, H.; Lindh, R.; de Meras, A. S.; Pedersen, T. B. In Linear-Scaling Techniques in Computational Chemistry and Physics: Methods and Applications; Zalesny, R., Papadopoulos, M. G., Mezey, P. G., Leszczynski, J., Eds.; Springer Netherlands: Dordrecht, 2011; pp 301-343.

(60) Harbrecht, H.; Peters, M.; Schneider, R. On the low-rank approximation by the pivoted Cholesky decomposition. Appl. Numer. Math. 2012, 62, 428 .

(61) Richard, R. M.; Lao, K. U.; Herbert, J. M. Understanding the many-body expansion for large systems. I. Precision considerations. J. Chem. Phys. 2014, 141, 014108.

(62) Wilson, H. F.; Gygi, F.; Galli, G. Efficient iterative method for calculations of dielectric matrices. Phys. Rev. B 2008, 78, 113303.

(63) Lu, D.; Gygi, F.; Galli, G. Dielectric properties of ice and liquid water from first-principles calculations. Phys. Rev. Lett. 2008, 100, 147601.

(64) Wilson, H. F.; Lu, D.; Gygi, F.; Galli, G. Iterative calculations of dielectric eigenvalue spectra. Phys. Rev. B 2009, 79, 245106.

(65) Nguyen, H.-V.; de Gironcoli, S. Efficient calculation of exact exchange and RPA correlation energies in the adiabatic-connection fluctuationdissipation theory. Phys. Rev. B 2009, 79, 205114.

(66) Baroni, S.; De Gironcoli, S.; Dal Corso, A.; Giannozzi, P. Phonons and related crystal properties from density-functional perturbation theory. Rev. Mod. Phys. 2001, 73, 515.

(67) Giannozzi, P.; Andreussi, O.; Brumme, T.; Bunau, O.; Nardelli, M. B.; Calandra, M.; Car, R.; Cavazzoni, C.; Ceresoli, D.; Cococcioni, M.; Colonna, N.; Carnimeo, I.; Corso, A. D.; de Gironcoli, S.; Delugas, P.; DiStasio, R. A.; Ferretti, A.; Floris, A.; Fratesi, G.; Fugallo, G.; Gebauer, R.; Gerstmann, U.; Giustino, F.; Gorni, T.; Jia, J.; Kawamura, M.; Ko, H.-Y.; Kokalj, A.; Kucukbenli, E.; Lazzeri, M.; Marsili, M.; Marzari, N.;
Mauri, F.; Nguyen, N. L.; Nguyen, H.-V.; de-la Roza, A. O.; Paulatto, L.; Ponce, S.; Rocca, D.; Sabatini, R.; Santra, B.; Schlipf, M.; Seitsonen, A. P.; Smogunov, A.; Timrov, I.; Thonhauser, T.; Umari, P.; Vast, N.; Wu, X.; Baroni, S. Advanced capabilities for materials modelling with Quantum ESPRESSO. J. Phys.: Condens. Matter 2017, 29, 465901.

(68) Hellgren, M.; Colonna, N.; De Gironcoli, S. Beyond the random phase approximation with a local exchange vertex. Phys. Rev. B 2018, 98, 045117.

(69) Govoni, M.; Galli, G. Large scale GW calculations. J. Chem. Theory Comput. 2015, 11, 2680.

(70) Saibaba, A. K.; Alexanderian, A.; Ipsen, I. C. Randomized matrix-free trace and log-determinant estimators. Numer. Math. 2017, 137, 353.

(71) Kaltak, M.; Klimes, J.; Kresse, G. Cubic scaling algorithm for the random phase approximation: Self-interstitials and vacancies in Si. Phys. Rev. B 2014, 90, 054115 .

(72) Takatsuka, A.; Ten-No, S.; Hackbusch, W. Minimax approximation for the decomposition of energy denominators in Laplace-transformed Moller-Plesset perturbation theories. J. Chem. Phys. 2008, 129, 044112.

(73) Haser, M.; Almlof, J. Laplace transform techniques in Moller-Plesset perturbation theory. $J$. Chem. Phys. 1992, 96, 489-494.

(74) Haser, M. Moller-Plesset (MP2) perturbation theory for large molecules. Theor. Chem. Acc. 1993, $87,147-173$.

(75) Ooura, T.; Mori, M. A robust double exponential formula for Fourier-type integrals. J. Comput. Appl. Math. 1999, 112, 229.

(76) Werner, H.-J.; Knowles, P. J.; Knizia, G.; Manby, F. R.; Schütz, M. Molpro: a generalpurpose quantum chemistry program package. WIREs Comput. Mol. Sci. 2012, 2, 242-253.

(77) Werner, H.-J.; Knowles, P. J.; Knizia, G.; Manby, F. R.; Schütz, M.; Celani, P.; Korona, T.; Lindh, R.; Mitrushenkov, A.; Rauhut, G.; Shamasundar, K. R.; Adler, T. B.; Amos, R. D.; Bernhardsson, A.; Berning, A.; Cooper, D. L.; Deegan, M. J. O.; Dobbyn, A. J.; Eckert, F.; Goll, E.; Hampel, C.; Hesselmann, A.; Hetzer, G.; Hrenar, T.; Jansen, G.; Köppl, C.; Liu, Y.; Lloyd, A. W.; Mata, R. A.; May, A. J.; McNicholas, S. J.; Meyer, W.; Mura, M. E.; Nicklass, A.; O'Neill, D. P.; Palmieri, P.; Peng, D.; Pflüger, K.; Pitzer, R.; Reiher, M.; Shiozaki, T.; Stoll, H.; Stone, A. J.; Tarroni, R.; 
Thorsteinsson, T.; Wang, M. MOLPRO, Version 2015.1, a Package of ab initio Programs. 2015, http://www.molpro.net.

(78) Hampel, C.; Peterson, K. A.; Werner, H.-J. A comparison of the efficiency and accuracy of the quadratic configuration interaction (QCISD), coupled cluster (CCSD), and Brueckner coupled cluster (BCCD) methods. Chem. Phys. Lett. 1992, $190,1-12$.

(79) Kresse, G.; Furthmüller, J. Efficient iterative schemes for ab initio total-energy calculations using a plane-wave basis set. Phys. Rev. B 1996, 54, 11169 .

(80) Halkier, A.; Klopper, W.; Helgaker, T.; Jorgensen, P.; Taylor, P. R. Basis set convergence of the interaction energy of hydrogen-bonded complexes. J. Chem. Phys. 1999, 111, 9157-9167.

(81) Schuchardt, K. L.; Didier, B. T.; Elsethagen, T.; Sun, L.; Gurumoorthi, V.; Chase, J.; Li, J.; Windus, T. L. Basis Set Exchange: A Community Database for Computational Sciences. J. Chem. Inf. Model. 2007, 47, 1045-1052.

(82) Adamo, C.; Barone, V. Toward reliable density functional methods without adjustable parameters: The PBE0 model. J. Chem. Phys. 1999, 110, 6158-6170.

(83) Hui, K.; Chai, J.-D. SCAN-based hybrid and double-hybrid density functionals from models without fitted parameters. J. Chem. Phys. 2016, $144,044114$.

(84) Patkowski, K.; Murdachaew, G.; Fou, C.-M.; Szalewicz, K. Accurate ab initio potential for argon dimer including highly repulsive region. Mol. Phys. 2005, 103, 2031-2045.

(85) Tucholska, A. M.; Modrzejewski, M.; Moszynski, R. Transition properties from the Hermitian formulation of the coupled cluster polarization propagator. J. Chem. Phys. 2014, 141, 124109.

(86) Tucholska, A. M.; Lesiuk, M.; Moszynski, R. Transition moments between excited electronic states from the Hermitian formulation of the coupled cluster quadratic response function. J. Chem. Phys. 2017, 146, 034108.

(87) Misquitta, A.; Podeszwa, R.; Jeziorski, B.; Szalewicz, K. Intermolecular potentials based on symmetry-adapted perturbation theory with dispersion energies from time-dependent densityfunctional calculations. J. Chem. Phys. 2005, 123, 214103.
(88) Kramida, A.; Yu. Ralchenko,; Reader, J.; and NIST ASD Team, NIST Atomic Spectra Database (ver. 5.6.1), [Online]. Available: https://physics.nist.gov/asd [Tue Aug 20 2019]. National Institute of Standards and Technology, Gaithersburg, MD., 2018.

(89) Chalasinski, G.; Szczesniak, M.; Cybulski, S. Calculations of nonadditive effects by means of supermolecular Moller-Plesset perturbation theory approach: $\mathrm{Ar}_{3}$ and $\mathrm{Ar}_{4}$. J. Chem. Phys. 1990, 92, 2481-2487.

(90) Chalasinski, G.; Szczesniak, M. M.; Kendall, R. A. Supermolecular approach to many-body dispersion interactions in weak van der Waals complexes: He, Ne, and Ar trimers. J. Chem. Phys. 1994, 101, 8860-8869. 